\title{
Review \\ The Medicinal Natural Products of Cannabis sativa Linn.: A Review
}

\author{
Anwuli Endurance Odieka ${ }^{1}\left(\mathbb{D}\right.$, Gloria Ukalina Obuzor ${ }^{2}$, Opeoluwa Oyehan Oyedeji ${ }^{3}\left(\mathbb{D}\right.$, Mavuto Gondwe $^{4}(\mathbb{O}$, \\ Yiseyon Sunday Hosu ${ }^{5}$ and Adebola Omowunmi Oyedeji ${ }^{1, *(1)}$
}

check for updates

Citation: Odieka, A.E.; Obuzor, G.U.; Oyedeji, O.O.; Gondwe, M.; Hosu, Y.S.; Oyedeji, A.O. The Medicinal Natural Products of Cannabis sativa Linn.: A Review. Molecules 2022, 27, 1689. https://doi.org/10.3390/ molecules27051689

Academic Editor: Francesco Paolo Busardò

Received: 31 December 2021 Accepted: 24 February 2022 Published: 4 March 2022

Publisher's Note: MDPI stays neutral with regard to jurisdictional claims in published maps and institutional affiliations.

Copyright: (C) 2022 by the authors. Licensee MDPI, Basel, Switzerland. This article is an open access article distributed under the terms and conditions of the Creative Commons Attribution (CC BY) license (https:// creativecommons.org/licenses/by/ $4.0 /)$.
1 Department of Chemical and Physical Sciences, Walter Sisulu University, Mthatha 5099, South Africa; endy_odieka@yahoo.com

2 Department of Pure and Industrial Chemistry, University of Port Harcourt, Port Harcourt 500004, Rivers State, Nigeria; gloria.obuzor@uniport.edu.ng

3 Department of Chemistry, Fort Hare University, Alice 5700, South Africa; ooyedeji@ufh.ac.za

4 Department of Human Biology, Walter Sisulu University, Mthatha 5099, South Africa; mgondwe@wsu.ac.za

5 Department of Economics and Business Sciences, Walter Sisulu University, Mthatha 5099, South Africa; yhosu@wsu.ac.za

* Correspondence: aoyedeji@wsu.ac.za; Tel.: +27-764-260-279

\begin{abstract}
Cannabis sativa is known among many cultures for its medicinal potential. Its complexity contributes to the historical application of various parts of the plant in ethno-medicines and pharmacotherapy. C. sativa has been used for the treatment of rheumatism, epilepsy, asthma, skin burns, pain, the management of sexually transmitted diseases, difficulties during child labor, postpartum hemorrhage, and gastrointestinal activity. However, the use of $C$. sativa is still limited, and it is illegal in most countries. Thus, this review aims to highlight the biological potential of the plant parts, as well as the techniques for the extraction, isolation, and characterization of C. sativa compounds. The plant produces a unique class of terpenophenolic compounds, called cannabinoids, as well as non-cannabinoid compounds. The exhaustive profiling of bioactive compounds and the chemical characterization and analysis of $C$. sativa compounds, which modern research has not yet fully achieved, is needed for the consistency, standardization, and the justified application of Cannabis sativa products for therapeutic purposes. Studies on the clinical relevance and applications of cannabinoids and non-cannabinoid phenols in the prevention and treatment of life-threatening diseases is indeed significant. Furthermore, psychoactive cannabinoids, when chemically standardized and administered under medical supervision, can be the legal answer to the use of C. sativa.
\end{abstract}

Keywords: medicinal plant; Cannabis sativa; phytochemicals; bioactivity; extraction methods; characterization

\section{Introduction}

The applications of plants as medicines predates human history. A medicinal plant refers to any plant which contains substances of therapeutic potential in one or more of its parts for the synthesis of plant-based drugs [1]. Active medicinal plant ingredients are referred to as bioactive phytochemicals. [2]. These bioactive compounds are believed to increase the ability of plants to survive or adapt to their surroundings [3] and are used as medicines, flavorings, and recreational drugs in humans. One notable medicinal plant that has continued to garner attention over the years, and in recent times, is Cannabis sativa.

Cannabis sativa L. is known for its medicinal uses since ancient times, because of its rich supply of phytochemicals [4], hence the quest for harnessing its pharmacological potential by scientists. The term "Cannabis" is used to define the products (drugs and essential oils) that are prepared or obtained from the annual herb C. sativa and its variants, which are of the family Cannabaceae [5]. The utilization of this multipurpose plant has been restrained for a long time because of the psychoactive effects of a specific cannabinoid 
( $\Delta^{9}$-tetrahydrocannabinol; $\mathrm{C}_{12} \mathrm{H}_{30} \mathrm{O}_{2}$ ) [6]. It was strongly prohibited in the twentieth century, and was removed from the British pharmacopeia. The plant was demonized due to its high abuse liability and supposedly insufficient health benefits [7]. Furthermore, due to the inability to prepare standardized preparations, and the diffusion of the recreational use of cannabis below therapeutic concentrations from the end of the 19th to the first half of the 20th century, the medical use of cannabis began to decline [7]. In 1937, the "Marihuana Tax Act", a federal legislation in the United States, functionally ended all medical uses of cannabis and was removed from the "National Formulary and Pharmacopoeia" in 1941 [7]. In 1961, cannabis resin, extracts, and tinctures were listed in the Schedule I of the single Convention on Narcotic Drugs, which prohibits the use, possession, production, manufacture, export, import, and trade of cannabis, except for medical and scientific purposes [7].

However, in multiple countries today, its cultivation and usage are regulated by laws [6,8]. Recent decriminalization policies and new scientific evidence have increased the interest in the medicinal potential of cannabis and have paved the way for the release of marketing authorizations for cannabis-based products [7]. In 1985, the United States Food and Drugs Administration (US FDA) reconsidered the medical use of cannabinoids, and approved Marinol (dronabinol) and Cesamet (nabilone), two synthetic analogues of tetrahydrocannabinol (THC), for the management of nausea and vomiting associated with cancer chemotherapy [7]. The Office of Medical Cannabis Research (OMC), a Dutch government agency in Europe, became the first organization to obtain the exclusive right to supply medical cannabis to research institutes and pharmacies, and, under the Single Convention on Narcotic Drugs of 1961, to import and export cannabis extracts and resin for medical purposes. Several medical cannabis products, all of which are dried female flowering tops, except Bediol (which is ground into small pieces for its easy manipulation by patients with spasticity), are exported by the OMC, with the proper licenses, to other member states of the European Union [7]. In Italy, the Military Pharmaceutical Chemical Works of Florence became the official national settlement for cultivating and manufacturing medical cannabis with a standard cannabinoid content [7,9]. The Italian Ministry of Health in November 2015, in a Ministerial Degree, authorized the indoor cultivation of cannabis flowering tops at a fixed temperature and at fixed light-dark cycles, leading to a standardized composition of different cannabinoids [9]. Two Italian products (FM1 and FM2) are currently available for consumers, and their use was approved for the treatment of chronic pain, neurological disorders, and other diseases resistant to standard therapies $[7,9,10]$. Non-psychoactive compounds found in C. sativa are associated with fewer side effects and can be used for several industrial applications [6]. The hemp stem supplies both cellulosic and woody fibers. The woody fibers are used for animal beddings, while the cellulosic fibers (bast fibers) are used as a substitute for fiberglass, and to produce bioplastics [4]. Its use as an anti-bacterial finishing agent and in functionalized textiles have also been reported [4]. The inflorescence was used, traditionally, for acute pain, insomnia, coughing, and wounds. The leaves were used for malaria, panting, roundworm, scorpion stings, hair loss, and the greying of hair. The stem bark was used for physical injury and strangury. Vaginal discharge, difficult births, strangury, the retention of the placenta, and physical injuries were treated using the roots [11]. In addition, Cannabis sativa contains essential oils of a high value, which can also improve the effectiveness of cannabinoids in pharmaceutical formulations [6]. 
Despite the influx of chemical-based medicines for treatments, the relevance of medicinal plants in drug development cannot be overemphasized. In recent years, commercial medicinal cannabis products with several variations in the phytocannabinoid content have been licensed and produced in Canada [7,8] and in several other countries. Several synthetic and standardized products are currently available on the market; however, patients' preferences lean towards herbal preparations, because they are easy to handle and selfadminister [7]. Thus, this review intends to highlight the phytochemicals present in the different plant parts, which potentiates their pharmacological activities, as well as the techniques for the extraction, isolation, and characterization of $C$. sativa compounds.

\section{Methods}

Literature on the published works of Cannabis sativa was obtained using electronic search engines, such as Google Scholar, the WSU online database (PubChem), and Science Direct. The keywords included, namely, Cannabis sativa, medicinal plants, Cannabis phytochemicals, ethnopharmacology bioactivity, and medicinal potentials, were used to source for data. An extensive review of the literature from 2011 through to 2021 (the last ten years) on Cannabis sativa L. was used to summarize its medicinal potential. Conversely, an emphasis will be placed on the isolation and characterization techniques from 1970 to 2021 to have a broadened view of the advancements in analytical techniques over the past years. Overall, twenty-nine (29) papers relating to the areas of our focus were chosen and were reviewed by all authors. The results from the search were carefully sorted, based on a general understanding, the review questions, and the related objectives.

\section{Origin and Botanical Description of $C$. sativa}

The genus name Cannabis means "cane-like" while sativa means "sown", which signifies that the plant is propagated from the seed and not from the roots [12]. It is believed to have originated in Asia and occurs widely in Africa [12,13]. Central and south-east Asia are the potential natural origins for the domestication of the Cannabis genus [14] and it is known by different common names in different languages (hemp, marihuana, kannabis sativa, ganja, bhang, and al-bhango) [15]. In South Africa, it is colloquially known, in Afrikaans, as "dagga"; in IsiXhoxa as "umfincafincane"; and in Isizulu as "umunyane" [16,17]. Taxonomically, Carl Linnaeus, a Swedish botanist, was the first to coin the name Cannabis sativa [18]. Other botanists stated that different types of Cannabis existed based on their size, shape, and resin content (breeding and selection). This review discusses, in particular, C. sativa.

The Cannabis phenotype (its observable traits or characteristics, such as its leaf shape and flower color) is based on two main factors: its genetic code (genotype) and the external environmental factors [19].

The roots are branched and are about 30-60 cm deep (Farag and Kayser, 2017) [12]. Cannabis inflorescence is made up of several flower heads found on long leafy stems from each leaf axil. A single brownish fruit, about $2-5 \mathrm{~mm}$ long, is produced per flower, and it contains a single seed tightly covered with a hard shell [12]. The fruit is propagated by bird and the seed germinates after 8-12 days [18]. The leaves, bracts, and stems of the plant are rich in trichrome, which are a diverse set of structures containing the secondary metabolites (phytocannabinoids and terpenoids) responsible for the defense, plant interactions, and typical smell [18]. Figure 1, below, shows the plant parts of C. sativa. 


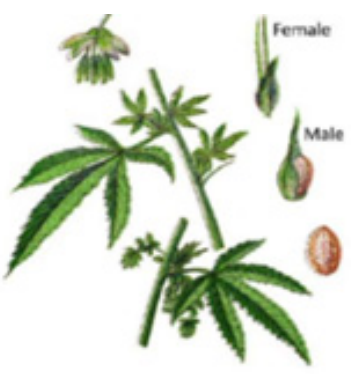

(a)

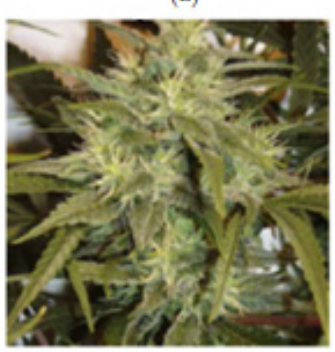

(c)

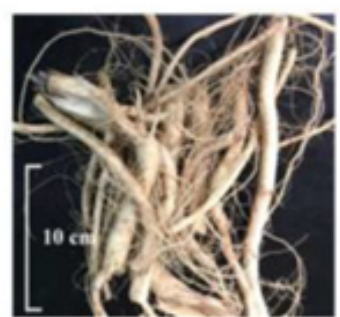

(b)

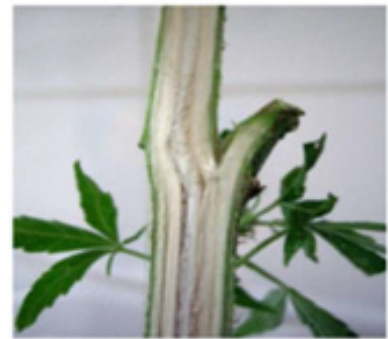

(d)

Figure 1. Cannabis plant parts. (a) Male and female Cannabis flowering parts with fresh leaf and seed. (b) Fresh Cannabis root. (c) Fresh Cannabis inflorescence (flower). (d) Fresh Cannabis stem bark.

\section{Phytochemistry of $C$. sativa}

\subsection{Chemical Profile of C. sativa}

Cannabis, as a herbal medicine, is a complex mixture of compounds, including cannabinoid phenols, non-cannabinoid phenols (stilbenoids, lignans, spiro-indans, and dihydrophenanthrenes), flavonoids, terpenoids, alcohols, aldehydes, n-alkanes, wax esters, steroids, and alkaloids $[6,8,11]$. Over 500 chemical compounds have been isolated from the cannabis plant and have been reported [13]. The several classes of secondary metabolites are present in different parts of the plant with a wide range of applications (nutraceuticals, cosmetics, aromatherapy, and pharmacotherapy) that are beneficial for humans. However, previous studies have focused mainly on the cannabinoids, $\Delta^{9}$-tetrahydrocannabinol $\left(\Delta^{9}\right.$-THC) and cannabidiol (CBD) in particular; hence, the female flower top is only harvested, while other parts of the plant are discarded [11].

Cannabinoids are a class of terpenophenolic compounds obtained by the alkylation of an alkyl-resorcinol with a monoterpene unit $[20,21]$. They feature alkyl resorcinol and monoterpene moieties in their molecules $[20,22]$. This specific chemical class in Cannabis is present in the glandular trichomes, which are abundant in the female flower as phytocannabinoid acids, and in the vegetable matrix as neutral phytocannabinoids $[6,13]$. They are biosynthesized by the alkylation of olivetolic acid with geranyl-pyrophosphate by a prenyltransferase to produce cannabigerolic acid (CBGA). Decarboxylation, a chemical reaction, converts the acidic forms ( $\Delta^{9}$ THCA, CBDA, CBCA, and CBGA) into their neutral forms, which are more active and efficient in terms of pharmacological activity $[8,23]$. To date, 125 cannabinoids have been identified and reported, in addition to five new cannabinoids reported in the past two years, 42 non-cannabinoid phenolics, 34 flavonoids, 120 terpenoids, 3 sterols, and 2 alkaloids $[8,11,13]$. Terpenoids are the second largest class of cannabis compounds and are responsible for their characteristic aroma [13]. Table 1 below summarizes the classes of compounds isolated from Cannabis sativa and the different plant parts in which they are present. 
Table 1. Chemical compounds Isolated from Cannabis sativa.

\begin{tabular}{|c|c|c|c|c|}
\hline $\mathrm{S} / \mathrm{N} *$ & Class of Compounds & Plant Part(s) & Isolated Compounds & References \\
\hline 1 & $\begin{array}{c}\text { Cannabinoids: } \\
\text {-Cannabinoid acids } \\
\text {-Neutral cannabinoids } \\
\text {-Cannabinoid } \\
\text { derivatives } \\
\text {-Cannabinoid } \\
\text { acid esters }\end{array}$ & $\begin{array}{l}\text { Leaves, flowers, } \\
\text { resin, stembarks, } \\
\text { and roots }\end{array}$ & $\begin{array}{c}\Delta^{9} \text {-tetrahydrocannabivarin, } \alpha / \beta \text {-fenchyl } \\
\Delta^{9} \text {-tetrahydrocannabinolate, } \\
\Delta^{9} \text {-tetrahydrocannabinol, } \alpha \text {-terpenyl } \\
(-)-\Delta^{9} \text {-trans-tetrahydrocannabinolate, } \\
\gamma \text {-eudesmyl } \\
(-) \Delta^{9} \text {-trans-tetrahydrocannabinolate, } \\
8 \alpha \text {-hydroxy-(-)- } \Delta^{9} \text {-trans- } \\
\text { tetrahydrocannabinol, } 8 b \text {-hydroxy-(-)- } \\
\Delta^{9} \text {-trans-tetrahydro cannabinol, } \\
\text { 8-oxo-(-)- } \Delta^{9} \text {-trans-tetrahydrocannabinol, } \\
\text { Cannabisol, } \\
(-)-\Delta^{9} \text {-trans-tetrahydrocannabiphorol, } \\
(-)-\Delta^{9} \text {-trans-tetrahydrocannabihexol, } \\
(-)-\Delta^{8} \text {-trans-tetrahydrocannabinol, } \Delta^{8} \text { - } \\
\text { trans-tetrahydrocannabinolic acid, } \\
\text { Cannabigerol, } \\
\text { 6,7-trans/cis-epoxycannabigerolic acid, } \\
\text { Sesquicannabigerol, Cannabigerolic acid, } \\
\text { Cannnabigerovarin, Cannabidiol, } \\
C_{4} \text {-Cannabidiol, Cannabidivarin, } \\
\text { Ca } \text {-tetrahydrocannabinol, } \\
\text { Cannabichromene, Cannabichromevarin, } \\
\text { Cannabichromanone, } \\
\text {-D } \text { D }_{4} \text {-acetoxycannabichromene, } \\
\text { Cannabicitran, Cannabiripsol, } \\
\text { Cannabicoumaronone, Cannabifuran, } \\
\text { Cannabielsoin, Cannabielsoic acid, } \\
\text { Cannabicyclol, Cannabinodiol, } \\
\text { bornyl/epi-bornyl- } \Delta^{9} \text { - } \\
\text { tetrahydrocannabinlate Cannabinol, } \\
\text { Cannabitriol, Cannabimovone, } \\
\text { and Cannabioxepane }\end{array}$ & {$[8,11,13,24-31]$} \\
\hline
\end{tabular}

\section{Non-Cannabinoid Constituents}

Dihydrostilbenes, Dihydrophenathrenes,

Cannabistilbene, Canniprene,

Cannithrene, Denbinobin,

Phloroglucinol $\beta$ - $D$-glucoside,

Cannabispiran, Cannabispirone, Cannabispirenone,

Non-cannabinoid phenol:
-Stilbenoids

-Spiro-indans

-Phenanthrenes

-Lignans,

lignanamides, and

phenolic amides
Leaves, flowers, stem, hemp pectin, resin, fruit, seed, and root
Cannabispirol, Cannabispirketal, $a$-cannabispiranol-4'-O- $\beta$-glucopyranose, prenylspirodienone, 4,5 -dihydroxy-2,3,6trimethoxy-9,10-dihydrophenanthrene, Cannabisin A-O

4,7-dimethoxy-1,2,5trihydroxyphenathrene, 5-methyl-4-pentyl-2,6,2trihydroxybiphenyl,

5-methyl-4-pentylbiphenyl-2,2,6-triol, $\mathrm{N}$-trans-coumaroyltyramine, $N$-trans-feruloyltyramine, Ntrans-caffeoyltyramine, 3,3'-demethylheliotropamide, and Grossamide. 
Table 1. Cont.

\begin{tabular}{|c|c|c|c|c|}
\hline $\mathbf{S} / \mathbf{N} *$ & Class of Compounds & Plant Part(s) & Isolated Compounds & References \\
\hline 3 & $\begin{array}{l}\text { Terpenoids (Terpenes): } \\
\text {-Monoterpenes } \\
\text {-Sesquiterpenes } \\
\text {-Diterpenes } \\
\text {-Triterpene, }\end{array}$ & $\begin{array}{l}\text { Essential oils of } \\
\text { fresh and dried } \\
\text { leaves, flowers, } \\
\text { stembarks, } \\
\text { and roots }\end{array}$ & $\begin{array}{c}\alpha \text {-pinene, } \beta \text {-pinene, linalool, linalool } \\
\text { oxide, myrcene, limonene, camphene, } \\
\alpha \text {-terpinene, } \gamma \text {-terpinene, } \alpha \text {-terpinolene, } \\
\alpha \text {-terpineol, terpinene-4-ol, sabinene, } \\
\text { sabinene hydrate, cis-sabinene hydrate, } \\
\alpha \text {-phellandrene, } \beta \text {-phellandrene, } \\
\text { 2-methyl-2-heptene-6-one, borneol, } \\
\text { piperitenone, geraniol, carvacrol, carvone, } \\
\text { cis-carveol, citronellol, bornyl acetate, } \\
\text { ipsdienol, germacrene-B, clovandiol, } \\
\alpha \text {-bisabolol, } \beta \text {-eudesmol, } \gamma \text {-eudesmol, } \\
\alpha \text {-caryophyllene, } \beta \text {-caryophyllene oxide, } \\
\alpha \text {-Humulene, Phytol, neophytadiene, } \\
\text { friedelin (friedelan-3-one), epifriedelanol, } \\
\beta \text {-amyrin, Vomifoliol, dihydrovomifoliol, } \\
\text { and dihydroactinidiolide } \\
\beta \text {-ionone }\end{array}$ & {$[8,11,13,28,29,35,36]$} \\
\hline 4 & $\begin{array}{c}\text { Flavonoids: } \\
\text {-Methylated } \\
\text {-Glycosylated (C or O } \\
\text { glycosides) } \\
\text {-Prenylated } \\
\text {-Geranylated }\end{array}$ & $\begin{array}{l}\text { Leaves, flowers, } \\
\text { seed, and fruit }\end{array}$ & $\begin{array}{l}\text { Orientin, Vitexin, Isovitexin, Apigenin, } \\
\text { Luteolin, Kaempferol, Quercetin, } \\
\text { Cytisoside, Cytisoside glucoside, } \\
\text { Canniflavone (Cannflavin), Naringenin, } \\
\text { and Naringin }\end{array}$ & $\begin{array}{c}{[8,11,13,28,29,35,37-} \\
39]\end{array}$ \\
\hline 5 & Sterols & $\begin{array}{l}\text { Stembarks, roots, } \\
\text { and leaves }\end{array}$ & $\begin{array}{c}\text { Campsterol, Stigmasterol, and } \\
\beta \text { - Sitosterol }\end{array}$ & {$[11,40]$} \\
\hline 7 & Alkaloids & $\begin{array}{c}\text { Roots, } \\
\text { leaves, stembark }\end{array}$ & $\begin{array}{l}\text { Cannabisativine } \\
\text { and Anhydrocannabisativine }\end{array}$ & {$[13,40]$} \\
\hline 8 & $\begin{array}{c}\text { Fatty acids: } \\
\text {-Saturated and } \\
\text { unsaturated fatty } \\
\text { acids and their esters }\end{array}$ & Seeds & $\begin{array}{l}\text { Roughanic acid, Stearidonic acid, } \\
\alpha \text {-linolenic acid, and oxylipins. }\end{array}$ & {$[8,28]$} \\
\hline 9 & $\begin{array}{l}\text { Hydrocarbons } \\
\text { (n-alkane) }\end{array}$ & - & $\Delta^{9}$-Tetrahydrocannabiorcolic acid & {$[8]$} \\
\hline
\end{tabular}

$* \mathrm{~S} / \mathrm{N}=$ Serial Number.

Figures 2-4 below show the structures of the different classes of bioactive compounds isolated from Cannabis sativa $[13,29,41]$.

\subsection{Extraction, Isolation, and Chemical Characterization of C. sativa}

Many methods have been reported for the extraction of Cannabis in the literature. These include direct maceration (DM), soxhlex extraction, ultrasound-assisted extraction (UAE), supercritical fluid extraction, and microwave-assisted extraction (MAE) [41]. However, two methods of extracting Cannabis are differentiated in the literature [41]. The first is the maceration of the plant material in an organic solvent (direct maceration) and the subsequent removal of the solvent by the concentration of the extract under reduced pressure [41]. The second is the innovative supercritical fluid extraction (SFE) method, which involves the use of pressurized solvents [41]. It is necessary for cannabinoid compounds to be extracted with organic solvents instead of water, because the active compounds are less soluble in polar solvents [41]. The most commonly used solvents are ethanol, ether, chloroform, and methanol [42]. When used for extraction, various compounds, including some undesired substances, dissolve together with the cannabinoids [42]. The high solvent power of ethanol for cannabinoid compounds is the reason why it is frequently used in home-made extracts of Cannabis [41]. However, non-desired compounds (chlorophyll, lipids, and waxy materials) are also extracted which, therefore, requires further steps to 
remove the co-extracted impurities for a high-purity medicinal product to be obtained [41]. A patent on the method for the isolation of herbal and cannabinoid medicinal extracts stated that the solubility of non-therapeutic substances (chlorophyll and waxy materials) is reduced when the solvent is selected from a group that includes acetonitrile, benzene, dichloromethane, diethyl ether, acetone, butanol, ethanol, chloroform, ethyl acetate, hexane, pentane, propanol, tetrahydrofuran, toluene, xylene, and various combinations of these solvents [41]. The International Conference on Harmonization (ICH) recommends the use of less toxic solvents in the manufacture of drug substances and dosage forms, and sets pharmaceutical limits for residual solvents in drug products [43]. Residual solvents pose risks to human health and are classified into three classes. Class 1 solvents (including carbon tetrachloride, benzene, and methyl chloroform) are regarded as human carcinogens and are environmentally hazardous [41]. Class 2 solvents include methanol and hexane, which are generally said to be limited, and they are possible causative agents of irreversible toxicity, such as neurotoxicity or teratogenicity [41]. Class 3 solvents (ethanol and ethyl acetate) are generally regarded as having a low toxic potential to humans [41]). Above all, ethanol is generally recognized as a safe (GRAS) solvent [41]. In a study by Brighenti et al., they compared the following four extraction techniques to obtain a high yield of medicinal cannabinoids: ultrasound-assisted extraction (UAE), microwave-assisted extraction (MAE), supercritical fluid extraction (SFE), and direct maceration (DM). They concluded that DM, with ethanol as the extraction solvent at room temperature for an overall time of $45 \mathrm{~min}$, is the best extraction technique (in terms of a high yield) for non-psychoactive cannabinoids from hemp [44].

Over the last decade, compounds in Cannabis have been identified, isolated, and determined by various chromatographic techniques with different spectroscopic detection methods. C. sativa samples are analyzed for both legal and medicinal purposes [41]. Nevertheless, the knowledge of their exact composition remains very significant. In 2009, recommended methods for the identification and analysis of cannabis products were released by the United Nations Office on Drugs and Crime [45]. One notable technique that has been employed in identifying the diverse composition of the compounds found is highperformance liquid chromatography (HPLC) [41]. Spectroscopic approaches or methods are based on the variable absorbance or redirection of electromagnetic (EM) radiation by chemical bonds, resulting in the radiation or transition of the sample's atoms to a higher energy state [46]. Some advantages are attributed to these spectroscopic methods, such as permitting spatial measurements of metabolites and offering a global metabolic fingerprint of a sample with rapid spectral acquisition [46]. Some of these approaches/methods include Fourier transform infrared spectroscopy (FTIR), nuclear magnetic resonance (NMR) spectroscopy, mass spectrometry (MS), HPLC, gas chromatography-mass spectrometry (GC-MS), and liquid chromatography-mass spectrometry (LS-MS) [41,46]. Taking into account the recommended methods and the mandatory requirement of the Ministerial decree to use only chromatographic techniques coupled with mass spectrometric detection, cannabinoid concentrations and its stability in cannabis tea and cannabis oil, prepared from standardized flowering tops obtained from the Military Pharmaceutical Chemical Works of Florence, were studied by Pacifici et al. using easy and fast ultra-high performance liquid chromatography-tandem mass spectrometry (UHPLC-MS/MS) [9,10]. Table 2 is a summary of the reported extraction solvents, as well as the identification, isolation, and characterization methods of Cannabis. 


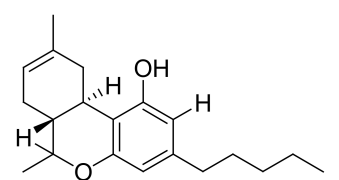

(a)<smiles>CCCCCc1cc(O)c(C2C=C(C)CCC2C(C)=O)c(O)c1C(=O)O</smiles>

(d)

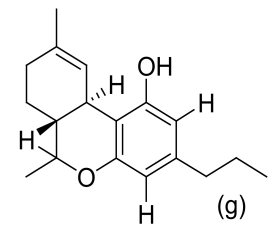<smiles>CCCCCc1cc2c(cc1CCCCC)-c1cc(C)ccc1C(C)(C)O2</smiles>

(j)<smiles>CCCCCc1cc(O)c2c(c1)OC(CC)C=C2</smiles>

(I)<smiles>CCCCCc1cc(O)c2c(c1)CC1CCC3C1C2C3(C)C</smiles>

(n)<smiles>CCCCCc1cc(O)c2c(c1)OC(C)[C@@H]1CCC(C)=C[C@H]21</smiles><smiles></smiles>

(e)<smiles>CCCCCc1cc2c(c(O)c1C(=O)O)[C@H]1C=C(C)CC[C@H]1C(C)O2</smiles><smiles>CCCc1cc(O)c(C2C=C(C)CCC2C(C)=O)c(O)c1</smiles><smiles>CCCCCc1cc(O)c(CC=C(C)C)c(O)c1C(=O)O</smiles>

(h)<smiles>CCCCCc1cc(O)c(CC=C(C)C)c(O)c1</smiles>

(i)<smiles>CCCCCc1cc2c(cc1C(=O)O)-c1cc(C)ccc1C(C)(C)O2</smiles>

(k)<smiles>CCCCCc1cc2c(c(O)c1C(=O)O)C=CC(CC)O2</smiles>

(m)<smiles>CCCCCc1cc2c(c(O)c1C(=O)O)C1C3C(CCC(C1(C)C)C3(C)C)C2</smiles>

(o)

Figure 2. Chemical structures of major cannabinoids; $\Delta^{8} \mathrm{THC}$, tetrahydrocannabinol (a); $\Delta^{9}-\mathrm{THC}$, tetrahydrocannabinol (b); THCA, tetrahydrocannabinolic acid (c); CBDA, cannabidiolic acid (d); CBD, cannabidiol (e); CBDV, cannabidivarin (f); THCV, tetrahydrocannabivarin (g); CBGA, cannabigerolic acid (h); CBG, cannabigerol (i); CBN, cannabinol (j); CBNA, cannabinolic acid (k); CBC, cannabichromene (1); CBCA, cannabichromenic acid (m); CBL, cannabicyclol (n); CBLA, cannabicyclolic acid (o). All structures drawn by Odieka, using ChemDraw Ultra 8.0. 
<smiles>C=CC(=C)CCC=C(C)C</smiles>

(a)<smiles>CC1=CC=C(C(C)C)CC1</smiles>

(e)<smiles>CC1=CCC2CC1C2(C)C</smiles>

(b)<smiles>C=CC(C)(O)CCC=C(C)C</smiles>

(c)<smiles>CC1=CCC(=C(C)C)CC1</smiles>

(g)<smiles>CC1=CCC(C(C)C)=CC1</smiles>

(d)<smiles>CC1=CCC(C(C)C)C=C1</smiles>

(f)<smiles>C=CC1(C)CCC(C(=C)C)CC1C(=C)C</smiles>

(j)<smiles>C=C1CC/C=C(\C)CCC2C1CC2(C)C</smiles>

(h)<smiles>CC1=CCCC=CC(C)(C)CC(C)=C1</smiles>

(i)<smiles>CC1CCC(C(C)(C)O)CC2=C1CCC2C</smiles><smiles>CC1C(=O)CCC2C1(C)CCC1C2(C)CCC2(C)C3CC(C)(C)CCC3(C)CCC12C</smiles><smiles>CC1C(O)CC[C@]2(C)C3CC[C@]4(C)C5CC(C)(C)CCC5(C)CCC4(C)C3CCC12C</smiles>

Figure 3. Chemical structures of some Cannabis sativa terpenes (Monoterpenes, Sesquiterpenes, and Triterpenoids); Mycene (a), $\alpha$-Pinene (b), D-Linalool (c), Limonene (d), $\alpha$-Terpinene (e), $\alpha$-Phellandrene (f), $\alpha$-Terpinolene $(\mathbf{g}), \beta$-Caryophyllene $(\mathbf{h}), \alpha$-Caryophyllene (i), $\beta$-Elemene $(\mathbf{j})$, Guaiol (k), Friedelin (1), and Epifriedelanol (m). All structures drawn by Odieka, using ChemDraw Ultra 8.0. 
<smiles>COc1cc(O)c2c(c1)CCC21CCC(=O)CC1</smiles>

(a)<smiles>COc1cc(O)c2c(c1)CCc1ccc(O)cc1-2</smiles>

(d)<smiles>COc1cc(O)c2c(c1)CCC21C=CC(=O)CC1</smiles><smiles>COc1cc(O)cc(CCc2ccc(OC)c(O)c2CC=C(C)C)c1</smiles>

(c)<smiles>COc1cc(O)c2c(c1)CCc1ccc(OC)c(O)c1-2</smiles>

(e)<smiles>COC1=CC2C=CC3=C(C(=O)C=C(OC)C3=O)C2C=C1</smiles>

(f)<smiles>COc1cc(-c2cc(=O)c3c(O)c(CC=C(C)C)c(O)cc3o2)ccc1O</smiles>

(g)<smiles>CCCCCCC(=O)CC1C=CCC2CC(=O)NCCCCNCCCN12</smiles>

(j)

(i)

Figure 4. Chemical structures of some non-cannabinoid phenols (Spirans, phenanthrenes, flavonoids, alkaloids); Cannabispiran (a), Cannabispirone (b), Canniprene (c), Cannithrene I (d), Cannithrene II (e), Debinobin (f), Canniflavin A (g), Canniflavin B (h), Cannabisativine (i), and Anhydrocannabisativine (j). All structures drawn by Odieka, using ChemDraw Ultra 8.0.

Table 2. Reported methods of identification, isolation, and characterization of C. sativa.

\begin{tabular}{|c|c|c|c|c|c|}
\hline Extraction Solvent(s) & Matrix and Species & $\begin{array}{c}\text { Identification, } \\
\text { Isolation, and } \\
\text { Purification Methods }\end{array}$ & $\begin{array}{l}\text { Elucidation/ } \\
\text { Analytical Techniques }\end{array}$ & Analytes & References \\
\hline \multicolumn{6}{|c|}{ Phenolic Cannabinoids } \\
\hline $\begin{array}{l}\text { Methanol/ } \\
\text { chloroform mixture. }\end{array}$ & C. sativa inflorescence & $\begin{array}{l}\text { Qualitative, } \\
\text { quantitative, and } \\
\text { comparative } \\
\text { derivatization study } \\
\text { of cannabinoids }\end{array}$ & Fast GC-MS & $\begin{array}{c}\text { CBDA, CBGA, CBG, CBD, THC, } \\
\Delta^{8} \text {-THC, CBC, THCA, THC }\end{array}$ & [47] \\
\hline $\begin{array}{l}\text { Supercritical } \\
\text { fluid extraction }\end{array}$ & $\begin{array}{l}\text { Plant biomass and } \\
\text { medicinal Cannabis resin }\end{array}$ & $\begin{array}{l}\text { Quantitative and } \\
\text { qualitative analysis } \\
\text { of cannabinoids }\end{array}$ & $\begin{array}{l}\text { UHPLC-DAD and } \\
\text { statistical analysis }\end{array}$ & $\begin{array}{l}\text { CBDA, THCA, CBD, CBN, } \\
\text { CBC, THC }\end{array}$ & [48] \\
\hline $\begin{array}{l}\text { Methanol/chloroform } \\
\text { solvent mixture }\end{array}$ & Cannabis flower samples & $\begin{array}{l}\text { Qualitative and } \\
\text { quantitative } \\
\text { measurement } \\
\text { of cannabinoids }\end{array}$ & HPLC-DAD & $\begin{array}{c}\Delta^{9}-\mathrm{THC}, \mathrm{CBD}, \mathrm{CBDA}, \mathrm{THCA}, \\
\mathrm{CBN}, \mathrm{CBG}, \mathrm{CBGA}, \Delta^{8}-\mathrm{THC}\end{array}$ & [49] \\
\hline $\begin{array}{l}\text { Ethanol/ } \\
\text { ethanolic extracts }\end{array}$ & $\begin{array}{l}\text { (i) Lebanese C. sativa } \\
\text { (ii) Cannabis } \\
\text { (iii) Decarbo-xylated } \\
\text { hemp leaves }\end{array}$ & $\begin{array}{l}\text { (i) Purified by } \\
\text { counter-current } \\
\text { distribution and silica } \\
\text { gel chromatography } \\
\text { (ii) Florisil and silica gel } \\
\text { column chromatography }\end{array}$ & $\begin{array}{l}\text { GCMS, IR, and }{ }^{1} \mathrm{H} \\
\text { NMR comparison with } \\
\text { an authentic sample }\end{array}$ & $\begin{array}{c}\text { Cannabielsoin, (+)-trans-CBT and } \\
(-) \text {-trans } \\
\text {-CBT-OEt-C }(\text { Cannibitriol }), \\
\text { Cannabicitran, and } \\
\text { Monomethylether of CBD }\end{array}$ & {$[13,50-52]$} \\
\hline
\end{tabular}


Table 2. Cont.

\begin{tabular}{|c|c|c|c|c|c|}
\hline Extraction Solvent(s) & Matrix and Species & $\begin{array}{c}\text { Identification, } \\
\text { Isolation, and } \\
\text { Purification Methods }\end{array}$ & $\begin{array}{c}\text { Elucidation/ } \\
\text { Analytical Techniques }\end{array}$ & Analytes & References \\
\hline $\begin{array}{c}\text { Sequential extraction } \\
\text { (hexanes, } \mathrm{CH}_{2} \mathrm{Cl}_{2} \\
\text { EtOAc, } \mathrm{EtOH}, \\
\text { EtOH/ } \mathrm{H}_{2} \mathrm{O} \text {, and } \mathrm{H}_{2} \mathrm{O} \text { ) }\end{array}$ & $\begin{array}{l}\text { (i) Bud and leaves of } \\
\text { high-potency variety of } \\
\text { C. sativa }\end{array}$ & $\begin{array}{c}\text { (i) Silica gel VLC, } \\
\text { C }_{18 \text {-solid phase }} \\
\text { extraction (SPE), and } \\
\text { HPLC } \\
\text { (ii) VLC } \\
\text { chromatography of } \\
\text { hexane extract, TLC, } \\
\text { flash silica gel, } \\
\text { Sephadex LH-20 } \\
\text { chromatography, and } \\
\text { semipreparative } \\
\text { reversed-phase (RP) } \\
\text { and chiral HPLC }\end{array}$ & $\begin{array}{l}\text { HRESIMS, 1D and 2D } \\
\text { NMR, GC-MS }\end{array}$ & 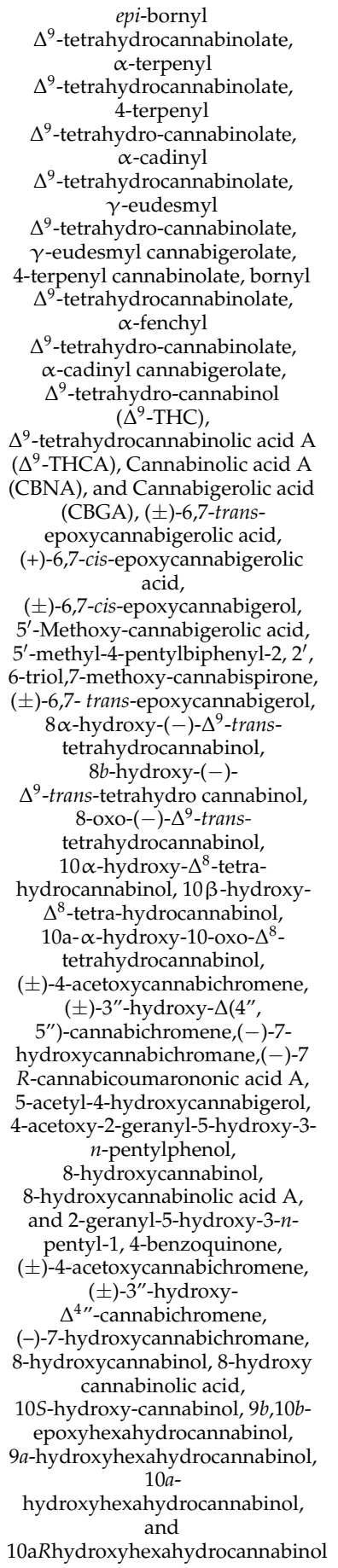 & {$[31,53-55]$} \\
\hline
\end{tabular}


Table 2. Cont.

\begin{tabular}{|c|c|c|c|c|c|}
\hline Extraction Solvent(s) & Matrix and Species & $\begin{array}{c}\text { Identification, } \\
\text { Isolation, and } \\
\text { Purification Methods }\end{array}$ & $\begin{array}{c}\text { Elucidation/ } \\
\text { Analytical Techniques }\end{array}$ & Analytes & References \\
\hline Hexane extract & $\begin{array}{c}\text { (i) C. sativa } \\
\text { (air-dried and } \\
\text { powdered buds) } \\
\text { (ii) high-potency variety } \\
\text { of C. sativa } \\
\text { (iii) Illicit Cannabis } \\
\text { samples } \\
\text { (iv) C. sativa } \\
\text { inflorescence (strain } \\
\text { CINRO) } \\
\text { (v) Lebanese C. sativa } \\
\text { (vi) Hemp } \\
\text { (vii) Nepalese and } \\
\text { Brazilian C. Sativa. }\end{array}$ & $\begin{array}{c}\text { (i) Column } \\
\text { chromatography using } \\
\text { silica or alumina, TLC, } \\
\text { then fractional } \\
\text { distillation and } \\
\text { preparative } \mathrm{C}_{18} \mathrm{HPLC} \\
\text { (ii) VLC (vacuum liquid } \\
\text { chromatography) silica } \\
\text { gel column } \\
\text { chromatography, } \mathrm{C}_{18} \\
\text { HPLC and chiral HPLC } \\
\text { (iii) Flash silica gel } \\
\text { chromatography } \\
\text { (iv) Florisil } \\
\text { column chromatography }\end{array}$ & $\begin{array}{c}{ }^{1} \mathrm{H} \text { NMR, }{ }^{13} \text { C NMR (2D } \\
\text { NMR) HRESIMS, } \\
\text { circular dichroism (CD), } \\
\text { UV, LC-HRMS, MS /MS, } \\
\text { GC-MS, and } \\
\text { confirmation by } \\
\text { phytochemical } \\
\text { transformations. }\end{array}$ & $\begin{array}{c}\Delta^{9} \text {-THC, } \Delta^{9} \text {-THC aldehyde, } \\
\text { Cannabinoid esters, Cannabisol, } \\
\Delta^{9} \text {-trans- } \\
\text { tetrahydrocannabiphorol, } \\
\Delta^{9} \text {-trans-tetrahydrocannabihexol, } \\
\text { Cannabidiorcol, Cannabidihexol } \\
\text { (CBDH) and Cannabidiphorol } \\
\text { (CBDP), Cannabitwinol, } \\
\text { Cannabinodivirin and } \\
\text { cannabinodiol (CBND), } \\
\text { Cannabichromene (CBC), } \\
\text { 9a-hydroxyhexahydrocannabinol, } \\
\text { 7-oxo-9a-hydroxyhexa- } \\
\text { hydrocannabinol, } \\
\text { 10a- } \\
\text { hydroxyhexahydrocannabinol, } \\
\text { and 10a- } R \text { - } \\
\text { hydroxyhexahydrocannabinol }\end{array}$ & {$[53,56-60]$} \\
\hline Ethyl acetate extracts & $\begin{array}{c}\text { Cannabis resins, } \\
\text { tinctures, and leaves }\end{array}$ & - & $\begin{array}{c}\text { GC-MS and } \\
\text { GC-FID analysis }\end{array}$ & $\Delta^{9}-\mathrm{THC}$ and $\Delta^{9}-\mathrm{THCA}$ & {$[61]$} \\
\hline Petroleum ether & $\begin{array}{l}\text { (i) Cannabis tincture of } \\
\text { Pakistani origin } \\
\text { (ii) Brazilian C. sativa } \\
\text { (iii) Cannabis leaves and } \\
\text { flowers (Maryland and } \\
\text { Czechoslovakian origin) } \\
\text { (iv) Congo C. sativa } \\
\text { (v) Hashish and } \\
\text { Cannabis sativa }\end{array}$ & $\begin{array}{l}\text { (i) Silicic acid column } \\
\text { chromatography } \\
\text { (ii) Silica gel and Florisil } \\
\text { chromatography, } \\
\text { preparative TLC }\end{array}$ & $\begin{array}{l}\text { IR, NMR, MS, GC-MS } \\
\text { confirmed by synthesis }\end{array}$ & $\begin{array}{c}\Delta^{9}-\mathrm{THCV}, \Delta^{9}-\mathrm{THCO} \text { or } \Delta^{9}-\mathrm{THC}, \\
\Delta^{8}-\mathrm{THC}\left(\Delta^{8}-\mathrm{THCA}\right), \\
\text { Cannabielsoin acid A (CBEAA,), } \\
\text { Cannabielsoin acid B, and } \\
\text { Cannabicyclovarin (CBLV) }\end{array}$ & [61-67] \\
\hline Benzene & $\begin{array}{l}\text { (i) Fresh C. sativa leaves } \\
\text { from Thailand } \\
\text { (ii) Fresh tops and } \\
\text { leaves of C. sativa } \\
\text { (iii) Hemp }\end{array}$ & $\begin{array}{c}\text { Polyamide and silica gel } \\
\text { column } \\
\text { chromatography }\end{array}$ & $\begin{array}{l}\text { IR, UV, NMR, and } \\
\text { comparing UV } \\
\text { spectrum with that } \\
\text { of derivatives }\end{array}$ & $\begin{array}{c}\Delta^{9} \text {-THCVA, CBDV, THCV, CBCV, } \\
\text { Cannabigerovarin CBGV, } \\
\text { cannabigerovarinic acid } \\
\text { (CBGVA), CBDA, } \\
\text { cannabidivarinic acid (CBDVA), } \\
\text { Cannabicyclolic acid (CBLA), and } \\
\text { Cannabichromenic acid (CBCA) }\end{array}$ & {$[49,68,69]$} \\
\hline Acetone extract & $\begin{array}{l}\text { (i) Leaves of C. sativa } \\
\text { (Mexican strain) } \\
\text { (ii) Wax of } \\
\text { decarboxylated aerial } \\
\text { parts of C. sativa (Carma } \\
\text { strain) } \\
\text { (iii) Cannabis } \\
\text { variety (carmagnola) }\end{array}$ & $\begin{array}{l}\text { (i) Silica gel column } \\
\text { chromatography } \\
\text { (ii) Silica and alumina } \\
\text { column } \\
\text { chromatography, } \\
\text { followed by normal } \\
\text { phase (NP)-HPLC. } \\
\text { (iii). Flash } \\
\text { chromatography, over } \\
\text { reverse-phased } \mathrm{C}_{18} \\
\text { silica gel followed by } \\
\text { normal-phase HPLC }\end{array}$ & $\begin{array}{l}\text { FAB-MS, }{ }^{1} \mathrm{H}-\mathrm{NMR}, \\
\left.{ }^{13} \mathrm{C}-\mathrm{NMR}\right), \text { and ESI-MS } \\
\text { semisyn-thesis. }\end{array}$ & $\begin{array}{l}\text { Cannabigerolic acid (CBGA), } \\
\text { dihydroxycannabigerol } \\
\text { derivative (camagerol), } \\
\text { Sesquicannabi-gerol, } \\
\text { Cannabimovone, and } \\
\text { Cannabioxepane }\end{array}$ & [70-73] \\
\hline \multicolumn{6}{|c|}{ Essential/volatile oils } \\
\hline Methanol dilutions & $\begin{array}{c}\text { Cannabis sativa oil } \\
\text { samples }\end{array}$ & $\begin{array}{c}\text { Separation/quantitation } \\
\text { of cannabinoids }\end{array}$ & Fast-GC-FID & $\mathrm{CBD}, \mathrm{CBN}$, and THC & [74] \\
\hline Essential oil & $\begin{array}{l}\text { Fresh C. sativa } \mathrm{L} \text {. } \\
\quad \text { from India }\end{array}$ & $\begin{array}{l}\text { Fractional distillation } \\
\text { and chromatography } \\
\text { over alumina. }\end{array}$ & $\begin{array}{c}\text { GC-MS and } \\
\text { physico-chemical } \\
\text { analyses }\end{array}$ & $\begin{array}{c}\alpha \text {-terpinene, } \beta \text {-phellandrene, } \\
\gamma \text {-terpinene, } \alpha \text {-terpinolene, } \\
\alpha \text {-pinene, } \beta \text {-pinene, camphene, } \\
\text { linalool, } \alpha \text {-terpineol, } \\
\text { terpinene- } 4 \text {-ol, linalool oxide, and } \\
\text { sabinene hydrate }\end{array}$ & [13] \\
\hline Volatile/essential oils & $\begin{array}{l}\text { (i) Cannabis } \\
\text { (Dutch and Turkish) } \\
\text { (ii) Fresh leaves of } \\
\text { Cannabis sativa and } \\
\text { Cannabis indica }\end{array}$ & $\begin{array}{l}\text { (i) Hydrodistillation or } \\
\text { through nitrogen } \\
\text { extraction } \\
\text { (ii) Hydrodistillation, } \\
\text { steam distillation, and } \\
\text { supercritical } \\
\text { fluid extraction }\end{array}$ & $\begin{array}{l}\text { Capillary gas } \\
\text { chromato-graphy, } \\
\text { GC-MS analysis }\end{array}$ & $\begin{array}{c}\text { cis- } \beta \text {-ocimene, trans- } \beta \text {-ocimene, } \\
\alpha \text {-phellandrene, } \mathrm{D}_{3} \text {-carene, } \\
\Delta^{4} \text {-carene, sabinene and } \\
\alpha \text {-thujene, caryophyllene, } \\
\text { humulene, trans- } \beta \text {-bergamotene, } \\
\text { cis- } \beta \text {-farnesene, } \delta \text {-limonene, } \\
\text { carophyllene oxide, linalool, } \\
\text { trans- } \alpha \text { - bergamotene, cis- } \beta \\
\text {-farnesene, menthol, eucalyptol, } \\
\text { and Carvone. }\end{array}$ & {$[13,75]$} \\
\hline Essential oil & $\begin{array}{l}\text { Cannabis (marijuana } \\
\text { fresh and dried buds) }\end{array}$ & Steamdistillation & GC-MS and GC-FID & $\begin{array}{l}\text { Ipsdienol, cis-carveol, and } \\
\text { cis-sabinene hydrate }\end{array}$ & [76] \\
\hline
\end{tabular}


Table 2. Cont.

\begin{tabular}{|c|c|c|c|c|c|}
\hline Extraction Solvent(s) & Matrix and Species & $\begin{array}{l}\text { Identification, } \\
\text { Isolation, and } \\
\text { Purification Methods }\end{array}$ & $\begin{array}{c}\text { Elucidation/ } \\
\text { Analytical Techniques }\end{array}$ & Analytes & References \\
\hline Essential oil & C. sativa resin & $\begin{array}{l}\text { Minor terpenic } \\
\text { component analysis }\end{array}$ & $\begin{array}{l}\text { GC-MS and GC } \\
\text { retention time }\end{array}$ & $\begin{array}{c}\alpha \text {-gurjunene, } \alpha \text {-bisabolol, } \\
\alpha \text {-cedrene, } \alpha \text {-cubebene, } \\
\delta \text {-cadinene, epi- } \beta \text {-santalene, } \\
\text { farnesol, } \gamma \text {-cadinene, } \gamma \text {-elemene, } \\
\gamma \text {-eudesmol, guaiol, } \\
(\mathrm{E}, \mathrm{E}) \text { - } \alpha \text {-farnesene, } \\
(\mathrm{Z})-\beta \text {-farnesene, and } \\
\text { farnesyl acetone }\end{array}$ & [77] \\
\hline Essential oil & Cannabis & $\begin{array}{l}\text { Steam distillation and } \\
\text { silica } \\
\text { gel chromatography }\end{array}$ & GC, GC-MS & $\begin{array}{l}\text { eugenol, methyleugenol, } \\
\text { iso-eugenol, trans-anethol, and } \\
\text { cis-anethol (simple phenols) }\end{array}$ & [78] \\
\hline Essential oil & C. sativa & $\begin{array}{l}\text { Column } \\
\text { chromatography of the } \\
\text { essential oil }\end{array}$ & $\begin{array}{c}\text { GC and } \\
\text { GC-MS analyses }\end{array}$ & $\begin{array}{l}\text { Iso-caryophyllene, } \beta \text {-selinene, } \\
\text { selina-3,7(11)-diene, and } \\
\text { selina-4(14),7(11)-diene }\end{array}$ & {$[13,79]$} \\
\hline \multicolumn{6}{|c|}{ Non-cannabinoid phenols } \\
\hline $\begin{array}{l}\text { Ethanol/ } \\
\text { ethanolic extract }\end{array}$ & $\begin{array}{l}\text { (i) South African } \\
\text { Cannabis variant } \\
\text { (ii) Saudi Arabia } \\
\text { hashish } \\
\text { (iii) Leaves of C. sativa } \\
\text { (iv) High-potency } \\
\text { Cannabis variety grown } \\
\text { in Mississippi } \\
\text { (v) Hemp pectin } \\
\text { (vi). Cannabis roots } \\
\text { (vii) Roots, stem, and } \\
\text { leaves of a Mexican } \\
\text { variant of } \\
\text { Cannabis sativa }\end{array}$ & $\begin{array}{l}\text { (i) Partitioning and } \\
\text { chromatography on } \\
\text { silica and polyamide } \\
\text { columns } \\
\text { (ii) Normal and } \\
\text { reversed phase } \\
\text { chromatographic } \\
\text { techniques } \\
\text { (iii) Purification by } \\
\text { macro reticular resin, } \\
\text { silica gel column } \\
\text { chromatography, and } \\
\text { Sephadex-LH-20 } \\
\text { (iv) TLC, } \\
\text { chromatography over } \\
\text { alumina, and } \\
\text { recrystallization } \\
\text { (v) Partitioning and } \\
\text { TLC eluted with chloro- } \\
\text { form:acetone:ammonia } \\
\text { (1:1:1) } \\
\text { (vi) Series of acid-base } \\
\text { extractions and } \\
\text { silica-gel } \\
\text { chromatography } \\
\text { followed by } \\
\text { crystallization of the } \\
\text { alkaloid from acetone }\end{array}$ & $\begin{array}{l}\text { IR, GCMS, UV, 1D NMR } \\
\text { (1H NMR, 13C NMR) } \\
\text { and 2DNMR (COSY, } \\
\text { HSQC, HMBC, and } \\
\text { ROESY), ESI-MS, } \\
\text { comparison with } \\
\text { authentic samples, } \\
\text { X-ray crystallography, } \\
\text { and semi-synthesis }\end{array}$ & 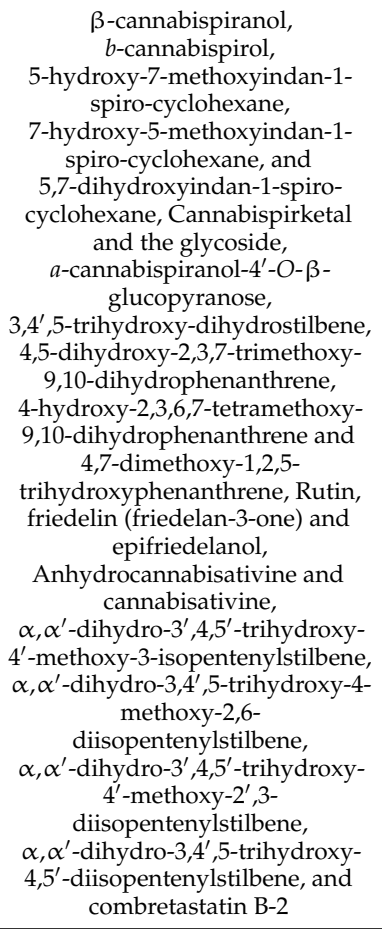 & $\begin{array}{c}{[32,33,55,80-} \\
84]\end{array}$ \\
\hline Benzene & $\begin{array}{l}\text { Dried leaves of } \\
\text { Japanese cannabis }\end{array}$ & $\begin{array}{l}\text { Chromatographed on a } \\
\text { polyamide column } \\
\text { followed by silica } \\
\text { gel chromatography }\end{array}$ & IR, ${ }^{1} \mathrm{H}$ NMR, MS, UV. & $\begin{array}{l}\text { Cannabispirol } \\
\text { and acetyl Cannabispirol }\end{array}$ & [85] \\
\hline Acetone & C. sativa (CARMA) & $\begin{array}{l}\text { Gravity column } \\
\text { chromatography on } \\
\text { silica gel and purified } \\
\text { by crystallization from } \\
\text { ether and HPLC }\end{array}$ & $\begin{array}{l}\text { Identified according to } \\
\text { its physical and } \\
\text { spectroscopic properties } \\
\text { and synthesis }\end{array}$ & $\begin{array}{c}\text { Debinoben } \\
\text { (1,4-phenanthrenequinone) }\end{array}$ & [86] \\
\hline
\end{tabular}


Table 2. Cont.

\begin{tabular}{|c|c|c|c|c|c|}
\hline Extraction Solvent(s) & Matrix and Species & $\begin{array}{c}\text { Identification, } \\
\text { Isolation, and } \\
\text { Purification Methods }\end{array}$ & $\begin{array}{c}\text { Elucidation/ } \\
\text { Analytical Techniques }\end{array}$ & Analytes & References \\
\hline $\begin{array}{c}\text { Sequential extraction } \\
\text { (Hexane, EtOAc, } \\
\mathrm{CH}_{2} \mathrm{Cl}_{2}, \text { EtOH, } \\
\text { EtOH } / \mathrm{H}_{2} \mathrm{O} \text {, and } \mathrm{H}_{2} \mathrm{O}\end{array}$ & $\begin{array}{l}\text { High-potency variety of } \\
\text { C. sativa (Mississippi) }\end{array}$ & $\begin{array}{l}\text { VLC, silica gel column } \\
\text { chromatography, and } \\
\text { RP-HPLC }\end{array}$ & $\begin{array}{l}\text { 1D and 2D NMR, } \\
\text { IR analysis }\end{array}$ & $\begin{array}{c}\text { acetoxy-6-geranyl-3- } n \text {-pentyl-1,4- } \\
\text { benzoquinone, } \\
\text { 4,5-dihydroxy-2,3,6-trimethoxy- } \\
\text { 9,10-dihydrophenanthrene, } \\
\text { 4-hydroxy-2,3,6,7-tetramethoxy- } \\
\text { 9,10-dihydrophenanthrene, } \\
\text { 4,7-dimethoxy-1,2,5- } \\
\text { trihydroxyphenanthrene, } \\
\text { Cannflavin C and } \\
\beta \text {-sitosteryl-3-O- } \beta \text {-D- } \\
\text { glucopyranoside-2'-O-palmitate, } \\
\alpha \text {-cannabispiranol, Chrysoeriol, } \\
\text { 6-prenylapigenin, and } \\
\text { Cannflavin A and } \\
\beta \text {-acetyl cannabispiranol }\end{array}$ & [55] \\
\hline Hexane extract & $\begin{array}{c}\text { Leaves of } \\
\text { Cannabis sativa }\end{array}$ & $\begin{array}{c}\text { Isolation by } \\
\text { normal-phase } \\
\text { chromatography } \\
\text { followed by } \mathrm{C}_{18} \text {-HPLC }\end{array}$ & $\begin{array}{c}\text { NMR and } \\
\text { ESI-MS analysis }\end{array}$ & $\begin{array}{l}\text { Prenylspirodinone and } \\
\text { 7-O-methyl-cannabispirone }\end{array}$ & {$[55,87]$} \\
\hline $\begin{array}{l}\text { Dichloro-methane } \\
\text { extract }\end{array}$ & $\begin{array}{l}\text { (i) Decarboxy-lated } \\
\text { C. sativa hemp } \\
\text { (ii). Thai Cannabis sativa } \\
\text { leaves } \\
\text { (iii) Panamanian variety } \\
\text { of cannabis }\end{array}$ & $\begin{array}{l}\mathrm{C}_{18} \text { flash } \\
\text { chromatography, } \\
\text { followed by silica gel } \\
\text { gravity column } \\
\text { chromatography } \\
\text { and HPLC }\end{array}$ & $\begin{array}{c}\text { HR-ESIMS and NMR } \\
\left({ }^{1} \mathrm{H},{ }^{13} \mathrm{C}, \mathrm{HSQC} \text {, and }\right. \\
\mathrm{HMBC}) \text { data, } \mathrm{X}-\text { ray } \\
\text { crystallography, and } \\
\text { confirmation } \\
\text { by hydrogenation }\end{array}$ & $\begin{array}{c}\text { Isocannabispiradienone and } \\
\text { a-Cannabispiranol, } \\
\text { Cannabispira-dienone, and } \\
\text { Cannabidihydro-phenanthrene } \\
\text { (Cannithrene1 and Cannithrene2) }\end{array}$ & {$[88,89]$} \\
\hline $\begin{array}{c}\text { Methanol/ } \\
\text { methanolic extract }\end{array}$ & $\begin{array}{c}\text { (i) Branches and leaves } \\
\text { of hemp } \\
\text { (ii) Pollen grains of } \\
\text { Mexican variety of } C \text {. } \\
\text { sativa } \\
\text { (iii) Dried leaves of } \\
\text { South African and } \\
\text { Indian cannabis sativa } \\
\text { (iv) Panamanian variant } \\
\text { of } C \text {. sativa } \\
\text { (v) Leaves and branches } \\
\text { of } C \text {. sativa }\end{array}$ & $\begin{array}{l}\text { (i) TLC, silica gel } \\
\text { column } \\
\text { chromatography, } \\
\text { normal-phase } \\
\text { preparative HPLC, and } \\
\text { Sephadex LH-20 } \\
\text { column } \\
\text { chromatography } \\
\text { (ii) Partitioning, silica } \\
\text { gel chromatography, } \\
\text { Sephadex LH-20 } \\
\text { chromatography, semi } \\
\text { preparative LC }\end{array}$ & $\begin{array}{l}\text { MS, } 1 \mathrm{D} \text { and 2D NMR, } \\
\text { UV experiments, IR, } \\
\text { X-ray crystallography } \\
\text { and confirmation by } \\
\text { total synthesis }\end{array}$ & $\begin{array}{c}\text { Rutin, } \\
\text { Quercetin-3-O- } \alpha-L \text {-rhamnoside, } \\
\text { kaempferol-3-O- } \alpha \text {-L-rhamnoside, } \\
\text { apigenin-7-O- } \alpha \text {-L-rhamnoside, } \\
\text { apigenin-7-O- } \beta-D \text { - } \\
\text { glucopyranoside, } \\
\text { luteolin-7-O- } \beta-D \text { - } \\
\text { glucopyranoside, } \\
\text { 1,3,6,7-tetrahydroxyl-2-C- } \beta-D \text { - } \\
\text { glucopyranosyl xanthone, vitexin, } \\
\text { orientin, apigenin-6,8-di-C- } \beta-D \text { - } \\
\text { glucopyranoside, } \\
\text { vitexin-2"-O- } \alpha-L-\text {-rhamnoside, } \\
\text { orientin-2"-O- } \beta-D- \\
\text { glucopyranoside, quebrachitol, } \\
\text { inositol and uracil, } \\
\text { kaempferol-3-O-sophoroside } \\
\text { (196) and } \\
\text { quercetin-3-O-sophoroside, } \\
\text { cannabispirone; } \\
\text { cannabispirenone, } \\
\text { Cannabispiran, Isocannabispiran } \\
\text { Canniprene, Cannabistilbene I } \\
\text { and Cannabistibene II, and } \\
\text { 2,3,5,6-tetramethoxy } \\
\text { 9,10-dihydrophenanthrenedione }\end{array}$ & [90-94] \\
\hline $\begin{array}{c}\text { Mixture of } \\
\text { hydro-alcoholic and } \\
\text { organic solvents }\end{array}$ & $\begin{array}{l}\text { C. sativa inflorescence } \\
\text { (Ferimon, Uso-31, } \\
\text { Felina } 32 \text {, and Fedora } \\
17 \text { cultivars) }\end{array}$ & $\begin{array}{l}\text { Metabolic and chemical } \\
\text { profiling to identify and } \\
\text { quantify compounds of } \\
\text { different classes }\end{array}$ & $\begin{array}{l}\text { NMR, GC-MS, UHPLC, } \\
\text { and HPLC-PDA }\end{array}$ & $\begin{array}{l}\text { Sugars, organic acids, amino } \\
\text { acids, cannabinoids, terpenoids, } \\
\text { phenols, tannins, flavonoids } \\
\text { (Quercetin, Naringenin, and } \\
\text { Naringin) and biogenic amines }\end{array}$ & {$[35,37]$} \\
\hline Diethyl ether & $\begin{array}{c}\text { Stem exudate } \\
\text { (greenhouse-grown } \\
\text { C. sativa) }\end{array}$ & $\begin{array}{l}\text { TLC and acid } \\
\text { hydrolysis of } \\
\text { the exudate }\end{array}$ & ${ }^{1} \mathrm{H}$ NMR and GC-MS & Phloroglucinol $\beta$ - $D$-glucoside & [95] \\
\hline
\end{tabular}

\subsection{Biological Evaluation/Potentials of C. sativa}

From the biological point of view, the psychoactive cannabinoids reported include $\Delta^{9}$ THC, cannabinol (CBN), and cannabinodiol (CBND), while cannabidiol (CBD) and other cannabinoids are non-psychoactive $[8,11]$. THC is the major psychoactive component and the toxicity of this metabolite of Cannabis is the most studied [11,28]. Its psychoactive component decreases in the order of inflorescence (the flower), leaves, stem, roots, and seeds, respectively [8]. The interest in the potential medical use of cannabis and cannabinoids rose significantly in the 1990s, following the discovery of the endocannabinoid (eCB) system in mammals [7]. The physiological effects of cannabinoids are exerted through 
various receptors, such as the cannabinoid receptors (CB1 and CB2), adrenergic receptors, and the recently discovered GPCRs $\left(\mathrm{GPR}_{55}, \mathrm{GPR}_{3}\right.$ and $\left.\mathrm{GPR}_{5}\right)$ [8]. Historically, each part of the Cannabis plant is indicated mostly for pain killing, inflammation, and for mental illnesses. For example, the Cannabis root has been recommended for treating fever, inflammation, gout, arthritis, and joint pain, as well as skin burns, hard tumors, postpartum hemorrhage, difficult child labor, sexually transmitted diseases, gastrointestinal activity, and infections [40]. Cannabis has also been used to treat asthma, epilepsy, fatigue, glaucoma, insomnia, nausea, pain, and rheumatism, as well as being used as appetite stimulant and a digestive aid $[7,11,13]$. Since concentrations above $0.05 \%$ are pharmacologically interesting, Cannabis inflorescence and leaf material may contain sufficient cannabinoids, mono- and sesquiterpenoids, and flavonoids for therapeutic applications [11]. Cannabis terpenoids and flavonoids, mainly myrcene, limonene, pinene, $\beta$-caryophyllene, and cannflavin A, act in synergy with cannabinoids to induce pharmacological effects [7]. It was proven that these compounds, which are synthetized in the aerial parts of the plant, enhance CBD's anti-inflammatory effects and antagonize THC dysphoric action [96]. Cannabidiol (CBD) and Cannabidavarin (CBDV) (neutral cannabinoids) have been reported to have the therapeutic potential for the treatment of epilepsy (focal seizures), as well as treating nausea and vomiting $[97,98]$. Conversely, THC and CBN have been found to be active in lowering intraocular pressure, and can be applied in all cases of glaucoma that are resistant to other therapies [9]. Cannflavin A and B are also notable flavonoids (prenylflavonoids) with medicinal potentials, such as their anti-inflammatory, anti-neoplastic, antioxidant, neuro-protective, anti-parasitic, and anti-viral effects [99]. Table 3, below, shows a summary of the reported bioactivities (biological potentials) of the bioactive compounds present in Cannabis sativa.

Table 3. Summary of reported bioactivities associated with isolated compounds and essential oils from Cannabis sativa.

\begin{tabular}{|c|c|c|}
\hline Isolated Bioactive Compound & Bioactivity/Uses & References \\
\hline Tetrahydrocannabinol THC & Antioxidant, anti-pruritic, and anti-inflammatory effects & {$[29,100]$} \\
\hline Cannabidiol CBD & $\begin{array}{c}\text { Anti-convulsive, anti-inflammatory, } \\
\text { immunosuppressive properties, antioxidant, and } \\
\text { anti-psychotic effects }\end{array}$ & [101-103] \\
\hline Cannabigerol CBG & $\begin{array}{c}\text { Anti-fungal effects, anti-cancer, anti-depressant, mild } \\
\text { anti-hypertensive agent, analgesic, and anti- } \\
\text { erythemic effects }\end{array}$ & {$[29,104]$} \\
\hline Cannabichromene $\mathrm{CBC}$ & Anti-inflammatory and analgesic & [29] \\
\hline Cannabinol CBN & $\begin{array}{l}\text { Sedative, anti-convulsant, anti-inflammatory, antibiotic, } \\
\text { and anti-MRSA activity }\end{array}$ & [29] \\
\hline Tetrahydrocannabivarin THCV & Anti-convulsant & [29] \\
\hline Tetrahydrocannabinolic acid THCA & $\begin{array}{l}\text { Immunomodulatory, anti-inflammatory, } \\
\text { neuroprotective, anti-neoplastic activity, and } \\
\text { antiemetic effects }\end{array}$ & {$[29,105]$} \\
\hline Cannabidavarin CBDV & $\begin{array}{l}\text { Anti-convulsant (anti-epileptic) properties and } \\
\text { anti-emetic properties }\end{array}$ & {$[106,107]$} \\
\hline Cannabidiolic acid CBDA & Anti-emetic effects & {$[104,108-110]$} \\
\hline$\beta$-Myrcene & Anti-inflammatory and analgesic sedative agent & {$[7,29,111]$} \\
\hline
\end{tabular}


Table 3. Cont.

\begin{tabular}{|c|c|c|}
\hline Isolated Bioactive Compound & Bioactivity/Uses & References \\
\hline$D$-Limonene & $\begin{array}{l}\text { Strongly anxiolytic, anti-depressant, antibiotic, and } \\
\text { anti-cancer agent }\end{array}$ & {$[7,29]$} \\
\hline$\beta$-Ocimene & $\begin{array}{l}\text { Anti-convulsant activity, anti-fungal activity, anti-tumor } \\
\text { activity, and pest resistance }\end{array}$ & {$[112,113]$} \\
\hline$\gamma$-Terpinene & $\begin{array}{l}\text { Anti-inflammatory activity, antioxidant, and } \\
\text { anti-proliferative activity }\end{array}$ & {$[114,115]$} \\
\hline$\alpha$-Terpinene & Antioxidant & [29] \\
\hline$\alpha$-Pinene & $\begin{array}{l}\text { Anti-inflammatory, bronchodilator, anti-microbial, } \\
\text { and anxiolytic effects }\end{array}$ & {$[7,11,29,116]$} \\
\hline Linalool & $\begin{array}{l}\text { Analgesic and anticonvulsant, anxiolytic, } \\
\text { anti-depressant, anti-glutamatergic, anti-leishmanial } \\
\text { activity, anticancer agent, anti-nociceptive, and } \\
\text { anti-depressant effects }\end{array}$ & {$[111,117-119]$} \\
\hline$\alpha$-Phellandrene & $\begin{array}{l}\text { Anti-nociceptive, anti-depressant, anti-arthritic and } \\
\text { allergic, and anti-hyperalgesic effects }\end{array}$ & [120-122] \\
\hline Terpinolene & $\begin{array}{l}\text { Anti-fungal and larvicidal, anti-nociceptive, } \\
\text { anti-inflammatory antioxidant, and anti-cancer effects }\end{array}$ & {$[123,124]$} \\
\hline$\beta$-Caryophyllene & $\begin{array}{c}\text { Cardio-protective, hepato-protective, gastro-protective, } \\
\text { neuro-protective, nephro-protective, antioxidant, } \\
\text { anti-inflammatory, anti-microbial, anti-pruritic, and } \\
\text { immunomodulatory activities }\end{array}$ & {$[7,125-127]$} \\
\hline Caryophyllene Oxide & $\begin{array}{l}\text { Anti-fungal, insecticidal/anti-feedant, and anti- } \\
\text { platelet effects }\end{array}$ & [29] \\
\hline$\beta$-Elemene & Anti-cancer and anti-tumor & [128] \\
\hline Guaiol & $\begin{array}{l}\text { Anti-inflammatory, antioxidant, anti-cancer } \\
\text { anti-rheumatic, antiseptic, diaphoretic, diuretic, and } \\
\text { laxative effects }\end{array}$ & {$[29,129]$} \\
\hline Friedelin & $\begin{array}{l}\text { Anti-inflammatory, anti-pyretic, and anti- } \\
\text { tuberculosis agent }\end{array}$ & {$[130,131]$} \\
\hline Epifriedelanol & Antioxidant & [132] \\
\hline Cannflavin A and B & $\begin{array}{l}\text { Anti-inflammatory, neoplastic, antioxidant, } \\
\text { neuroprotective, anti-parasitic, and anti-viral agent }\end{array}$ & {$[4,7,29,99]$} \\
\hline Apigenin & $\begin{array}{c}\text { Anxiolytic and estrogenic properties, anti-tumor, } \\
\text { antioxidant, anti-inflammatory, anti-osteoporosis, and } \\
\text { immune regulation effects }\end{array}$ & {$[4,133]$} \\
\hline Vitexin and Isovitexin & $\begin{array}{l}\text { Antioxidant, anti-cancer, anti-inflammatory, } \\
\text { anti-diabetic, anti-microbial, anti-viral, } \\
\text { anti-hyperalgesic, and neuroprotective effects }\end{array}$ & [134] \\
\hline Quercetin & $\begin{array}{l}\text { Anti-cancer/anti-proliferator, antioxidative/anti-aging, } \\
\text { anti-viral, anti-inflammatory, cardio-protective, } \\
\text { skin-protective, anti-coagulant, and anti-platelet effects }\end{array}$ & [135] \\
\hline Luteolin & $\begin{array}{l}\text { Neuroprotective effects, anti-inflammatory, and } \\
\text { antioxidant effects }\end{array}$ & [136] \\
\hline Lignans & $\begin{array}{c}\text { Antioxidant, anti-viral, anti-diabetic, anti-tumorigenic, } \\
\text { and anti-obesity activities }\end{array}$ & [4] \\
\hline
\end{tabular}


Cannabis female flowering tops can be simply administered through commercially available vaporizers (e.g., Micro Vape, G Pen Herbal Vaporizer, and Volcano), buccal sprays (e.g., Sativex), oral capsules (e.g., Cannador), decoctions, or oils [7]. Only cannabis use through oral or inhalatory administration is allowed. Smoking reduces the bioavailability of cannabis ingredients by $40 \%$, and its complete combustion can cause lung diseases and airway obstructions [7]. Homemade decoctions and pharmacy oils are currently the most widespread cannabis formulations in Europe, making the standardization of preparation difficult [7]. Cannabis pharmacological action is dose-dependent and can induce many adverse effects (AEs), principally related to THC, due to unintentional overdosing [7]. The typical symptoms of cannabis acute intoxication that have been reported are dizziness, confusion, tachycardia, postural hypotension, dysphoria, panic depression, hallucinations, allergic reactions, vomiting, and diarrhea $[7,137,138]$. Furthermore, withdrawal symptoms, such as irritability, aggression, anxiety, insomnia, decreased appetite, tremors, sweating, and headaches may appear after the abrupt cessation of the long-term administration of high doses of cannabis [7]. According to the ICH efficacy and safety guidelines, it is recommended to start with low doses and increase quantities after a satisfactory period of clinic evaluation, depending on the pharmacological effects and the possible adverse effects [139].

In the current COVID-19 pandemic, scientists are repurposing medicines (identifying new therapeutic use(s) of existing drugs) known for their biological potential (anti-viral or anti-inflammatory properties) to tackle the global issue and similar future viruses [140]. They have hypothesized that CBD could be used as an anti-viral agent [141] or antiinflammatory [142,143] tool, or to inhibit pulmonary fibrosis in COVID-19 patients [144]. In addition, the known growing evidence of the anxiolytic effects of CBD have also been hypothesized to be used as a therapeutic option to treat long-lasting COVID-19-related anxiety and PTSD [145], which is likely to be a significant issue of the pandemic.

\section{Conclusions}

With the recent evaluation, acceptance, and legalization of Cannabis products for therapeutic purposes, researchers, particularly in the field of natural products, are challenged to improve and standardize the extraction and characterization of the bioactive compounds from Cannabis sativa. Despite various reports of its economic and therapeutic values, it is legal in a handful of jurisdictions (Uruguay, Canada, some US states, and parts of Africa). Presently, Cannabis remains illegal in several countries. This review summarized the biological potential and the techniques for the extraction, isolation, and characterization of Cannabis sativa compounds, and it describes the effectiveness of the various parts of the herb in pharmacotherapy. The usage of $C$. sativa roots and stem barks in present-day medical research, and the development of new Cannabis-based medicines or products, in contrast to the flowering part only, is highly recommended because they can be exploited for medicine and other uses. In addition, Cannabis-based pharmaceutical products must undergo long purification processes to eliminate unwanted components such as chlorophyll and residual organic solvents. The use of standardized reagents is also very crucial in the analytical studies of $C$. sativa. Furthermore, future research should seek to clarify the factors responsible for the complexity of C. sativa extracts in terms of their chemical compositions, the physical properties of their active ingredients, and their liability to photochemical oxidation.

Author Contributions: Conceptualization, A.E.O., A.O.O., G.U.O., O.O.O., M.G. and Y.S.H.; methodology, A.E.O., G.U.O., O.O.O., M.G., Y.S.H. and A.O.O.; validation, A.E.O., M.G., Y.S.H. and A.O.O.; data curation, A.E.O.; writing-original draft preparation, AEO; writing-review and editing, A.E.O., A.O.O., O.O.O., G.U.O., M.G. and Y.S.H.; supervision, A.O.O., G.U.O., O.O.O., M.G. and Y.S.H.; project administration, A.O.O.; funding acquisition, A.E.O. and A.O.O. All authors have read and agreed to the published version of the manuscript. 
Funding: This research was funded by National Research Foundation (NRF), grant numbers 130205 and 137963.

Data Availability Statement: Not applicable.

Acknowledgments: The authors acknowledge the Directorate of Research and Innovation, Walter Sisulu University (WSU), South Africa and Govan Mbeki Research and Development Center, University of Fort Hare, South Africa.

Conflicts of Interest: The authors declare no conflict of interest.

\section{References}

1. Sofowora, A.; Ogunbodede, E.; Onayade, A. The role and place of medicinal plants in the strategies for disease prevention. Afr. J. Tradit. Complement. Altern. Med. 2013, 10, 210-229. [CrossRef]

2. Choudhary, N.; Siddiqui, M.; Bi, S.; Khatoon, S. Variation in preliminary phytochemicals screening of Cannabis sativa L. leaf, stem and root. Int. J. Pharmacogn. 2014, 1, 516-519.

3. Bandar, H.; Hijazi, A.; Rammal, H.; Hachem, A.; Saad, Z.; Badran, B. Techniques for the extraction of bioactive compounds from Lebanese Urtica Dioica. Am. J. Phytomed. Clin. Ther. 2013, 1, 507-513.

4. Andre, C.M.; Hausman, J.F.; Guerriero, G. Cannabis sativa: The plant of the thousand and one molecules. Front. Plant Sci. 2016, 7, 19. [CrossRef] [PubMed]

5. Elsohly, M.A.; Radwan, M.M.; Gul, W.; Chandra, S.; Galal, A. Phytochemistry of Cannabis sativa L. Phytocannabinoids 2017, 103, 1-36.

6. Baldino, L.; Scognamiglio, M.; Reverchon, E. Supercritical fluid technologies applied to the extraction of compounds of industrial interest from Cannabis sativa L. and to their pharmaceutical formulations: A review. J. Pharm. Fluids 2020, 165, 104960. [CrossRef]

7. Brunetti, P.; Pichini, S.; Pacifici, R.; Busardò, F.P.; del Rio, A. Herbal preparations of medical cannabis: A vademecum for prescribing doctors. Medicina 2020, 56, 237. [CrossRef]

8. Lewis, M.M.; Yang, Y.; Wasilewski, E.; Clarke, H.A.; Kotra, L.P. Chemical profiling of medical Cannabis extracts. ACS Omega 2017, 2, 6091-6103. [CrossRef] [PubMed]

9. Pacifici, R.; Marchei, E.; Salvatore, F.; Guandalini, L.; Busardò, F.P.; Pichini, S. Evaluation of cannabinoids concentration and stability in standardized preparations of cannabis tea and cannabis oil by ultra-high performance liquid chromatography tandem mass spectrometry. Clin. Chem. Lab. Med. 2017, 55, 1555-1563. [CrossRef]

10. Pacifici, R.; Marchei, E.; Salvatore, F.; Guandalini, L.; Busardò, F.P.; Pichini, S. Evaluation of long-term stability of cannabinoids in standardized preparations of cannabis flowering tops and cannabis oil by ultra-high-performance liquid chromatography tandem mass spectrometry. Clin. Chem. Lab. Med. 2018, 56, 94-96. [CrossRef] [PubMed]

11. Jin, D.; Dai, K.; Xie, Z.; Chen, J. Secondary metabolites profiled in cannabis inflorescences, leaves, stem barks, and roots for medicinal purposes. Sci. Rep. 2020, 10, 1-14.

12. Farag, S.; Kayser, O. The Cannabis plant: Botanical aspects. In Handbook of Cannabis and Related Pathologies; Academic Press: Cambridge, MA, USA, 2017; pp. 3-12.

13. Radwan, M.M.; Chandra, S.; Gul, S.; Elsohly, M.A. Cannabinoids, Phenolics, Terpenes and Alkaloids of Cannabis. Molecules 2021, 26, 2774. [CrossRef]

14. Stevens, C.J.; Murphy, C.; Roberts, R.; Lucas, L.; Silva, F.; Fuller, D.Q. Between China and South Asia: A Middle Asian corridor of crop dispersal and agricultural innovation in the bronze age. Holocene 2016, 26, 1541-1555. [CrossRef] [PubMed]

15. Chandra, S.; Lata, H.; Khan, I.A.; Elsohly, M.A. Cannabis sativa L.: Botany and horticulture. In Cannabis sativa L.-Botany and Biotechnology; Springer: Berlin/Heidelberg, Germany, 2017; pp. 79-100.

16. Duvall, C.S. A brief agricultural history of Cannabis in Africa, from prehistory to canna-colony. Echogéo 2019, 48, 1-25. [CrossRef]

17. Nsuala, B.N.; Enslin, G.; Viljoen, A. "Wild Cannabis": A review of the traditional use and phytochemistry of Leonotis leonurus. J. Ethnopharmacol. 2015, 174, 520-539. [CrossRef] [PubMed]

18. Bonini, S.A.; Premoli, M.; Tambaro, S.; Kumar, A.; Maccarinelli, G.; Memo, M.; Mastinu, A. Cannabis sativa: A comprehensive ethnopharmacological review of a medicinal plant with a long history. J. Ethnopharmacol. 2018, 227, 300-315. [CrossRef] [PubMed]

19. Dutch Passion. Understanding Cannabis Phenotypes, Genotypes and Chemotypes. 2020. Available online: https://dutch-passion.com/en/blog/understanding-cannabis-phenotypes-genotypes-and-chemotypes-n980 (accessed on 19 February 2022).

20. Hanuš, L.O.; Meyer, S.M.; Muñoz, E.; Taglialatela-Scafati, O.; Appendino, G. Phytocannabinoids: A unified critical inventory. Nat. Prod. Rep. 2016, 33, 1357-1392. [CrossRef] [PubMed]

21. Appendino, G.; Chianese, G.; Taglialatela-Scafati, O. Cannabinoids: Occurrence and medicinal chemistry. Curr. Med. Chem. 2011, 18, 1085-1099. [CrossRef] [PubMed]

22. Hill, A.J.; Williams, C.M.; Whalley, B.J.; Stephens, G.J. Phytocannabinoids as novel therapeutic agents in CNS disorders. Pharmacol. Ther. 2012, 133, 79-97. [CrossRef]

23. Lewis-Bakker, M.M.; Yang, Y.; Vyawahare, R.; Kotra, L.P. Extractions of medical Cannabis cultivars and the role of decarboxylation in optimal receptor responses. Cannabis Cannabinoid Res. 2019, 4, 183-194. [CrossRef] [PubMed] 
24. Elsohly, M.; Gul, W. Constituents of Cannabis sativa. Handb. Cannabis 2014, 3, 1093.

25. Elzinga, S.; Fischedick, J.; Podkolinski, R.; Raber, J.C. Cannabinoids and terpenes as chemotaxonomic markers in Cannabis. Nat. Prod. Chem. Res. 2015, 3, 1-9.

26. Hazekamp, A.; Tejkalová, K.; Papadimitriou, S. Cannabis: From cultivar to chemovar II-A metabolomics approach to cannabis classification. Cannabis Cannabinoid Res. 2016, 1, 202-215.

27. Lynch, R.C.; Vergara, D.; Tittes, S.; White, K.; Schwartz, C.; Gibbs, M.J.; Ruthenburg, T.C.; Decesare, K.; Land, D.P.; Kane, N.C. Genomic and chemical diversity in Cannabis. Crit. Rev. Plant Sci. 2016, 35, 349-363. [CrossRef]

28. Pollastro, F.; Minassi, A.; Fresu, L.G. Cannabis phenolics and their bioactivities. Curr. Med. Chem. 2018, 25, 1160-1185.

29. Russo, E.B.; Marcu, J. Cannabis pharmacology: The usual suspects and a few promising leads. Adv. Pharmacol. 2017, 80, 67-134.

30. Upton, R.; Elsohly, M. (Eds.) Cannabis Inflorescence: Cannabis Spp.; Standards of Identity, Analysis, and Quality Control; American Herbal Pharmacopoeia: Scotts Valley, CA, USA, 2014.

31. Radwan, M.M.; Elsohly, M.A.; El-Alfy, A.T.; Ahmed, S.A.; Slade, D.; Husni, A.S.; Manly, S.P.; Wilson, L.; Seale, S.; Cutler, S.J.; et al. Isolation and pharmacological evaluation of minor cannabinoids from high-potency Cannabis sativa. J. Nat. Prod. 2015, 78, 1271-1276. [CrossRef] [PubMed]

32. Guo, T.; Liu, Q.; Hou, P.; Li, F.; Guo, S.; Song, W.; Zhang, H.; Liu, X.; Zhang, S.; Zhang, J.; et al. Stilbenoids and cannabinoids from the leaves of Cannabis sativa f. sativa with potential reverse cholesterol transport activity. Food Funct. 2018, 9, 6608-6617. [CrossRef]

33. Guo, T.T.; Zhang, J.C.; Zhang, H.; Liu, Q.C.; Zhao, Y.; Hou, Y.F.; Bai, L.; Zhang, L.; Liu, X.Q.; Liu, X.Y.; et al. Bioactive spirans and other constituents from the leaves of Cannabis sativa f. sativa. J. Asian Nat. Prod. Res. 2017, 19, 793-802. [CrossRef] [PubMed]

34. Yan, X.; Tang, J.; dos Santos Passos, C.; Nurisso, A.; Simoes-Pires, C.A.; Ji, M.; Lou, H.; Fan, P. Characterization of lignanamides from hemp (Cannabis sativa L.) seed and their antioxidant and acetylcholinesterase inhibitory activities. J. Agric. Food Chem. 2015, 63, 10611-10619. [CrossRef]

35. Ingallina, C.; Sobolev, A.P.; Circi, S.; Spano, M.; Fraschetti, C.; Filippi, A.; di Sotto, A.; di Giacomo, S.; Mazzoccanti, G.; Gasparrini, F.; et al. Cannabis sativa L. inflorescences from monoecious cultivars grown in central Italy: An untargeted chemical characterization from early flowering to ripening. Molecules 2020, 25, 1908. [CrossRef] [PubMed]

36. Menghini, L.; Ferrante, C.; Carradori, S.; D’antonio, M.; Orlando, G.; Cairone, F.; Cesa, S.; Filippi, A.; Fraschetti, C.; Zengin, G.; et al. Chemical and bioinformatics analyses of the anti-leishmanial and anti-oxidant activities of hemp essential oil. Biomolecules 2021, 11, 272. [CrossRef] [PubMed]

37. Di Giacomo, V.; Recinella, L.; Chiavaroli, A.; Orlando, G.; Cataldi, A.; Rapino, M.; di Valerio, V.; Politi, M.; Antolini, M.D.; Acquaviva, A.; et al. Metabolomic profile and antioxidant/anti-inflammatory effects of industrial hemp water extract in fibroblasts, keratinocytes and isolated mouse skin specimens. Antioxidants 2021, 10, 44. [CrossRef] [PubMed]

38. Weston-Green, K. The united chemicals of Cannabis: Beneficial effects of cannabis phytochemicals on the brain and cognition. In Recent Advances in Cannabinoid Research; IntechOpen: London, UK, 2018.

39. Smeriglio, A.; Galati, E.M.; Monforte, M.T.; Lanuzza, F.; D’angelo, V.; Circosta, C. Polyphenolic Compounds and antioxidant activity of cold-pressed seed oil from finola cultivar of Cannabis sativa L. Phytother. Res. 2016, 30, 1298-1307. [CrossRef]

40. Ryz, N.R.; Remillard, D.J.; Russo, E.B. Cannabis roots: A traditional therapy with future potential for treating inflammation and pain. Cannabis Cannabinoid Res. 2017, 2, 210-216. [CrossRef]

41. Ramirez, C.L.; Fanovich, M.A.; Churio, M.S. Cannabinoids: Extraction methods, analysis, and physicochemical characterization. Stud. Nat. Prod. Chem. 2019, 66, 143-173.

42. Płotka-Wasylka, J.; Rutkowska, M.; Owczarek, K.; Tobiszewski, M.; Namieśnik, J. Extraction with environmentally friendly solvents. TrAC Trends Anal. Chem. 2017, 91, 12-25. [CrossRef]

43. International Conference on Harmonisation (ICH). International Council for Harmonisation of Technical Requirements for Pharmaceuticals for Human Use. ICH Harmonised Guideline. Impurities: Guideline for Residual solventsq3c(R6). 2016. Available online: https:/ / database.ich.org/sites/default/files/Q3C-R6_Guideline_ErrorCorrection_2019_0410_0.pdf (accessed on 20 January 2022).

44. Brighenti, V.; Pellati, F.; Steinbach, M.; Maran, D.; Benvenuti, S. Development of a new extraction technique and hplc method for the analysis of non-psychoactive cannabinoids in fibre-type Cannabis sativa L.(hemp). J. Pharma. Biomed. Anal. 2017, 143, 228-236. [CrossRef] [PubMed]

45. Recommended Methods for the Identification and Analysis of Cannabis and Cannabis Products. 2009. Available online: http:/ / www.unodc.org/documents/scientific/ST-NAR-40-Ebook.pdf (accessed on 1 November 2021).

46. Allwood, J.W.; Ellis, D.I.; Goodacre, R. Metabolomic technologies and their application to the study of plants and plant-host interactions. Physiol. Plant. 2008, 132, 117-135. [CrossRef] [PubMed]

47. Cardenia, V.; Toschi, T.G.; Scappini, S.; Rubino, R.C.; Rodriguez-Estrada, M.T. Development and validation of a fast gas chromatography/mass spectrometry method for the determination of cannabinoids in Cannabis sativa L. J. Food Drug Anal. 2018, 26, 1283-1292. [CrossRef] [PubMed]

48. Rochfort, S.; Isbel, A.; Ezernieks, V.; Elkins, A.; Vincent, D.; Deseo, M.A.; Spangenberg, G.C. Utilisation of design of experiments approach to optimise supercritical fluid extraction of medicinal Cannabis. Sci. Rep. 2020, 10, 1-7. [CrossRef] [PubMed]

49. Patel, B.; Wene, D.; Fan, Z.T. Qualitative and quantitative measurement of cannabinoids in Cannabis using modified hplc/dad method. J. Pharma Biomed. Anal. 2017, 146, 15-23. [CrossRef] [PubMed] 
50. Bercht, C.; Lousberg, R.; Küppers, F.; Salemink, C.; Vree, T.; van Rossum, J. Cannabis: VII. Identification of cannabinol methyl ether from hashish. J. Chromatogr. Anal. 1973, 81, 163-166.

51. Elsohly, M.; El-Feraly, F.; Turner, C. Isolation and characterization of (+) cannabitriol and () 10 ethoxy 9 hydroxy delta $6 a$ tetrahydrocannabinol: Two new cannabinoids from Cannabis sativa L. extract. Lloydia 1977, 40, 275-280. [PubMed]

52. Shoyama, Y.; Kuboe, K.; Nishioka, I.; Yamauchi, T. Cannabidiol monomethyl ether. A new neutral cannabinoid. Chem. Pharm. Bull. 1972, 20, 2072. [CrossRef]

53. Ahmed, S.A.; Ross, S.A.; Slade, D.; Radwan, M.M.; Khan, I.A.; Elsohly, M.A. Minor oxygenated cannabinoids from high potency Cannabis sativa L. Phytochemistry 2015, 117, 194-199. [CrossRef] [PubMed]

54. Ahmed, S.A.; Ross, S.A.; Slade, D.; Radwan, M.M.; Zulfiqar, F.; Elsohly, M.A. Cannabinoid ester constituents from high-potency Cannabis sativa. J. Nat. Prod. 2008, 71, 536-542. [CrossRef] [PubMed]

55. Radwan, M.M.; Elsohly, M.A.; Slade, D.; Ahmed, S.A.; Wilson, L.; El-Alfy, A.T.; Khan, I.A.; Ross, S.A. Non-cannabinoid constituents from a high potency Cannabis sativa variety. Phytochemistry 2008, 69, 2627-2633. [CrossRef] [PubMed]

56. Chianese, G.; Lopatriello, A.; Schiano-Moriello, A.; Caprioglio, D.; Mattoteia, D.; Benetti, E.; Ciceri, D.; Arnoldi, L.; de Combarieu, E.; Vitale, R.M.; et al. Cannabitwinol, a dimeric phytocannabinoid from hemp, Cannabis sativa L.; is a selective thermo-TRP modulator. J. Nat. Prod. 2020, 83, 2727-2736. [CrossRef] [PubMed]

57. Citti, C.; Linciano, P.; Russo, F.; Luongo, L.; Iannotta, M.; Maione, S.; Laganà, A.; Capriotti, A.L.; Forni, F.; Vandelli, M.A.; et al. A novel phytocannabinoid isolated from Cannabis sativa $\mathrm{L}$. with an in vivo cannabimimetic activity higher than $\Delta^{9}$-: $\Delta^{9}$-tetrahydrocannabiphorol. Sci. Rep. 2019, 9, 20335. [CrossRef] [PubMed]

58. Elsohly, M.A.; Ross, S.A. Method of Preparing Delta-9-Tetrahydrocannabinol. U.S. Patent US6365416B1, 4 March 2002.

59. Linciano, P.; Citti, C.; Russo, F.; Tolomeo, F.; Laganà, A.; Capriotti, A.L.; Luongo, L.; Iannotta, M.; Belardo, C.; Maione, S.; et al. Identification of a new cannabidiol n-hexyl homolog in a medicinal cannabis variety with an antinociceptive activity in mice: Cannabidihexol. Sci. Rep. 2020, 10, 22019. [CrossRef] [PubMed]

60. Zulfiqar, F.; Ross, S.A.; Slade, D.; Ahmed, S.A.; Radwan, M.M.; Ali, Z.; Khan, I.A.; Elsohly, M.A. Cannabisol, a novel $\Delta 9-T H C$ dimer possessing a unique methylene bridge, isolated from Cannabis sativa. Tetrahedron Lett. 2012, 53, 3560-3562. [CrossRef] [PubMed]

61. Harvey, D. Characterization of the butyl homologues of D-1-tetrahydrocannabinol, cannabinol and cannabidiol in samples of cannabis by combined gas chromotography and mass spectrometry. J. Pharm. Pharmacol. 1976, 28, 280-285. [CrossRef] [PubMed]

62. Archer, R.A.; Boyd, D.B.; Demarco, P.V.; Tyminski, I.J.; Allinger, N. Structural studies of cannabinoids. Theoretical and proton magnetic resonance analysis. J. Am. Chem. Soc. 1970, 92, 5200-5206. [CrossRef]

63. Elsohly, M.A.; Slade, D. Chemical constituents of marijuana: The complex mixture of natural cannabinoids. Life Sci. 2005, 78, 539-548. [CrossRef] [PubMed]

64. Gill, E.W. Propyl homologue of tetrahydrocannabinol: Its isolation from Cannabis, properties, and synthesis. J. Chem. Soc. C 1971, 3, 579-582. [CrossRef]

65. Krejcí, Z.; Šantavý, F. Isolation of two new cannabinoid acids from Cannabis sativa L. of Czechoslovak origin. Acta Univ. Olomuc. Fac. Med. 1975, 74, 161-166.

66. Shani, A.; Mechoulam, R. Cannabielsoic acids: Isolation and synthesis by a novel oxidative cyclization. Tetrahedron 1974, 30, 2437-2446. [CrossRef]

67. Vree, T.; Breimer, D.; van Ginneken, C.A.M.; van Rossum, J.M. Identification in hashish of tetrahydrocannabinol, cannabidiol and cannabinol analogues with a methyl side-chain. J. Pharm. Pharmacol. 1972, 24, 7-12. [CrossRef] [PubMed]

68. Shoyama, Y.; Hirano, H.; Makino, H.; Umekita, N.; Nishioka, I. Cannabis. X. The isolation and structures of four new propyl cannabinoid acids, tetrahydrocannabivarinic acid, cannabidivarinic acid, cannabichromevarinic acid and cannabigerovarinic acid, from Thai Cannabis,'meao variant'. Chem. Pharma. Bull. 1977, 25, 2306-2311. [CrossRef]

69. Shoyama, Y.; Hirano, H.; Oda, M.; Somehara, T.; Nishioka, I. Cannabichromevarin and cannabigerovarin, two new propyl homologues of cannabichromene and cannabigerol. Chem. Pharm. Bull. 1975, 23, 1894-1895. [CrossRef]

70. Appendino, G.; Giana, A.; Gibbons, S.; Maffei, M.; Gnavi, G.; Grassi, G.; Sterner, O. A polar cannabinoid from Cannabis sativa Var. Carma. Nat. Prod. Commun. 2008, 3, 1934578X0800301207. [CrossRef]

71. Pagani, A.; Scala, F.; Chianese, G.; Grassi, G.; Appendino, G.; Taglialatela-Scafati, O. Cannabioxepane, a novel tetracyclic cannabinoid from hemp, Cannabis sativa L. Tetrahedron 2011, 67, 3369-3373. [CrossRef]

72. Taglialatela-Scafati, O.; Pagani, A.; Scala, F.; de Petrocellis, L.; di Marzo, V.; Grassi, G.; Appendino, G. Cannabimovone, a cannabinoid with a rearranged terpenoid skeleton from hemp. Eur. J. Org. Chem. 2010, 2010, 2023. [CrossRef]

73. Taura, F.; Morimoto, S.; Shoyama, Y. Cannabinerolic acid, a cannabinoid from Cannabis sativa. Phytochemistry 1995, 39, 457-458. [CrossRef]

74. Borges, G.R.; Birk, L.; Scheid, C.; Morés, L.; Carasek, E.; Kitamura, R.O.S.; Roveri, F.L.; Eller, S.; de Oliveira Merib, J.; de Oliveira, T.F. Simple and straightforward analysis of cannabinoids in medicinal products by fast-GC-FID. Forensic Toxicol. 2020, 38, 531-535. [CrossRef]

75. Naz, S.; Hanif, M.A.; Bhatti, H.N.; Ansari, T.M. Impact of supercritical fluid extraction and traditional distillation on the isolation of aromatic compounds from Cannabis indica and Cannabis sativa. J. Essent. Oil Bear. Plants 2017, 20, 175-184. [CrossRef]

76. Ross, S.A.; Elsohly, M.A. The volatile oil composition of fresh and air-dried buds of Cannabis sativa. J. Nat. Prod. 1996, 59, 49-51. [CrossRef] [PubMed] 
77. Strömberg, L. Minor Components of Cannabis Resin: IV. Mass spectrometric data and gas chromatographic retention times of terpenic components with retention times shorter than that of cannabidiol. J. Chromatogr. A 1974, 96, 99-114. [CrossRef]

78. Turner, C.E.; Elsohly, M.A.; Boeren, E.G. Constituents of Cannabis sativa L. XVII. A review of the natural constituents. J. Nat. Prod. 1980, 43, 169-234. [CrossRef]

79. Hendriks, H.; Malingré, T.M.; Batterman, S.; Bos, R. Alkanes of the essential oil of Cannabis sativa. Phytochemistry 1977, 16, 719-721. [CrossRef]

80. El-Feraly, F.S.; El-Sherei, M.M.; Al-Muhtadi, F.J. Spiro-indans from Cannabis sativa. Phytochemistry 1986, 25, 1992-1994. [CrossRef]

81. Chen, B.; Cai, G.; Yuan, Y.; Li, T.; He, Q.; He, J.F. Chemical constituents in hemp pectin I. Chin. J. Exp. Tradit. Med. Form. 2012, $18,98-100$

82. Boeren, E.; Elsohly, M.; Turner, C.; Salemink, C. B-Cannabispiranol: A new non-cannabinoid phenol from Cannabis sativa L. Experiential 1977, 33, 848. [CrossRef] [PubMed]

83. Slatkin, D.J.; Doorenbos, N.J.; Harris, L.S.; Masoud, A.N.; Quimby, M.W.; Schiff, P.L. Chemical constituents of Cannabis sativa L. root. J. Pharm. Sci. 1971, 60, 1891-1892. [CrossRef]

84. Latter, H.L.; Abraham, D.J.; Turner, C.E.; Knapp, J.E.; Schiff, P.L., Jr.; Slatkin, D.J. Cannabisativine, a new alkaloid from Cannabis sativa L. root. Tetrahedron Lett. 1975, 16, 2815-2818. [CrossRef]

85. Shoyama, Y.; Nishioka, I. Cannabis. XIII. Two new spiro-compounds, cannabispirol and acetyl cannabispirol. Chem. Pharm. Bull. 1978, 26, 3641-3646. [CrossRef]

86. Sánchez-Duffhues, G.; Calzado, M.A.; de Vinuesa, A.G.; Caballero, F.J.; Ech-Chahad, A.; Appendino, G.; Krohn, K.; Fiebich, B.L.; Muñoz, E. Denbinobin, a naturally occurring 1, 4-phenanthrenequinone, inhibits HIV-1 replication through an NF- $\mathrm{B}$-dependent pathway. Biochem. Pharmacol. 2008, 76, 1240-1250. [CrossRef] [PubMed]

87. Nalli, Y.; Arora, P.; Riyaz-Ul-Hassan, S.; Ali, A. Chemical investigation of Cannabis sativa leading to the discovery of a prenylspirodinone with anti-microbial potential. Tetrahedron Lett. 2018, 59, 2470-2472. [CrossRef]

88. Crombie, L.; Crombie, W.M.L. Natural products of Thailand high $\Delta$ 1-THC-strain Cannabis. the bibenzyl-spirandihydrophenanthrene group: Relations with cannabinoids and canniflavones. J. Chem. Soc. Perkin Trans. 1982, 1, 1455-1466. [CrossRef]

89. Ross, S.; Elsohly, M.A. Constituents of Cannabis sativa L. XXVIII. A review of the natural constituents. Zagazig J. Pharm. Sci. 1995, 4,1-10. [CrossRef]

90. Elsohly, H.N.; Ma, G.E.; Turner, C.E.; Elsohly, M.A. Constituents of Cannabis sativa, XXV. Isolation of two new dihydrostilbenes from a panamanian variant. J. Nat. Prod. 1984, 47, 445-452. [CrossRef] [PubMed]

91. Ottersen, T.; Aasen, A.; El-Feraly, F.S.; Turner, C.E. X-ray structure of cannabispiran: A novel Cannabis constituent. Chem. Commun. 1976, 15, 580-581. [CrossRef]

92. Ross, S.A.; Elsohly, M.A.; Sultana, G.N.; Mehmedic, Z.; Hossain, C.F.; Chandra, S. Flavonoid glycosides and cannabinoids from the pollen of Cannabis sativa L. Phytochem. Anal. Int. J. Plant Chem. Biochem. Tech. 2005, 16, 45-48. [CrossRef] [PubMed]

93. Cheng, L.; Kong, D.; Hu, G.; Li, H. A new 9, 10-dihydrophenanthrenedione from Cannabis sativa. Chem. Nat. Compd. 2010, 46, 710-712. [CrossRef]

94. Bercht, C.; van Dongen, J.; Heerma, W.; Lousberg, R.C.; Küppers, F. Cannabispirone and cannabispirenone, two naturally occurring spiro-compounds. Tetrahedron 1976, 32, 2939-2943. [CrossRef]

95. Hammond, C.T.; Mahlberg, P.G. Phloroglucinol glucoside as a natural constituent of Cannabis sativa. Phytochemistry 1994, 37, 755-756. [CrossRef]

96. Namdar, D.; Voet, H.; Ajjampura, V.; Nadarajan, S.; Mayzlish-Gati, E.; Mazuz, M.; Shalev, N.; Koltai, H. Terpenoids and phytocannabinoids co-produced in Cannabis sativa strains show specific interaction for ellcytotoxic activity. Molecules 2019, 24, 3031. [CrossRef]

97. Whalley, B.; Stephens, G.; Williams, C.; Guy, G.; Wright, S.; Kikuchi, T.; GW Pharma Ltd.; Otsuka Pharmaceutical Co Ltd. Use of One or a Combination of Phyto-Cannabinoids in the Treatment of Epilepsy. U.S Patent 9,066,920, 30 June 2015.

98. Parker, L.; Rock, E.; Sticht, M.; Wills, K.; Limebeer, C.L. Cannabinoids suppress acute and anticipatory nausea in preclinical rat models of conditioned gaping. Pharm. Ther. 2015, 97, 559-561. [CrossRef] [PubMed]

99. Erridge, S.; Mangal, N.; Salazar, O.; Pacchetti, B.; Sodergren, M.H. Cannflavins-From plant to patient: A scoping review. Fitoterapia 2020, 146, 104712. [CrossRef] [PubMed]

100. Pacher, P.; Mechoulam, R. Is lipid signaling through cannabinoid 2 receptors part of a protective system? Prog. Lipid Res. 2011, 50, 193-211. [CrossRef] [PubMed]

101. Mcguire, P.; Robson, P.; Cubala, W.J.; Vasile, D.; Morrison, P.D.; Barron, R.; Taylor, A.; Wright, S.J. Cannabidiol (CBD) as an adjunctive therapy in schizophrenia: A multicenter randomized controlled trial. Am. J. Psychiatry 2018, 175, 225-231. [CrossRef] [PubMed]

102. Parker, L.A.; Rock, E.M.; Limebeer, C.L. Regulation of nausea and vomiting by cannabinoids. Br. J. Pharmacol. 2011, 163, 1411-1422. [CrossRef] [PubMed]

103. Yeshurun, M.; Shpilberg, O.; Herscovici, C.; Shargian, L.; Dreyer, J.; Peck, A.; Israeli, M.; Levy-Assaraf, M.; Gruenewald, T.; Mechoulam, R.; et al. Cannabidiol for the prevention of graft-versus-host-disease after allogeneic hematopoietic cell transplantation: Results of a phase II study. Biol. Blood Marrow Transpl. 2015, 21, 1770-1775. [CrossRef] [PubMed] 
104. Brierley, D.I.; Samuels, J.; Duncan, M.; Whalley, B.J.; Williams, C.M. Cannabigerol is a novel, well-tolerated appetite stimulant in pre-satiated rats. Psychopharmacology 2016, 233, 3603-3613. [CrossRef] [PubMed]

105. Rock, E.; Kopstick, R.L.; Limebeer, C.L.; Parker, L.A. Tetrahydrocannabinolic acid reduces nausea-induced conditioned gaping in rats and vomiting in S uncus murinus. Br. J. Pharmacol. 2013, 170, 641-648. [CrossRef]

106. Rock, E.M.; Sticht, M.A.; Parker, L.A. Effect of Phytocannabinoids on Nausea and Vomiting; Oxford University Press: Oxford, $\mathrm{UK}, 2014$

107. Tsien, R.; Whalley, B.J.; Devinsky, O. Cannabinoids and Epilepsy. Neurotherapeutics 2015, 12, 747-768.

108. Moreno-Sanz, G. Can you pass the acid test? critical review and novel therapeutic perspectives of $\Delta 9$-tetrahydrocannabinolic acid A. Cannabis Cannabinoid Res. 2016, 1, 124-130. [CrossRef] [PubMed]

109. Rock, E.; Parker, L.A. Effect of low doses of cannabidiolic acid and ondansetron on licl-induced conditioned gaping (a model of nausea-induced behaviour) in rats. Br. J. Pharmacol. 2013, 169, 685-692. [CrossRef] [PubMed]

110. Rock, E.M.; Connolly, C.; Limebeer, C.L.; Parker, L.A. Effect of combined oral doses of $\Delta$ 9-tetrahydrocannabinol (THC) and cannabidiolic acid (CBDA) on acute and anticipatory nausea in rat models. Psychopharmacology 2016, 233, 3353-3360. [CrossRef] [PubMed]

111. Russo, E.B. Taming THC: Potential cannabis synergy and phytocannabinoid-terpenoid entourage effects. Br. J. Pharmacol. 2011, 163, 1344-1364. [CrossRef] [PubMed]

112. Cascone, P.; Iodice, L.; Maffei, M.E.; Bossi, S.; Arimura, G.I.; Guerrieri, E. Tobacco overexpressing $\beta$-ocimene induces direct and indirect responses against aphids in receiver tomato plants. J. Plant Physiol. 2015, 173, 28-32. [CrossRef] [PubMed]

113. Bomfim, L.M.; Menezes, L.R.; Rodrigues, A.C.; Dias, R.B.; Gurgel Rocha, C.A.; Soares, M.B.; Neto, A.F.; Nascimento, M.P.; Campos, A.F.; Silva, L.C.; et al. Antitumour activity of the microencapsulation of Annona vepretorum essential oil. Basic Clin. Pharmacol. 2016, 118, 208-213. [CrossRef] [PubMed]

114. De Oliveira Ramalho, T.R.; de Oliveira, M.T.; de Araujo Lima, A.L.; Bezerra-Santos, C.R.; Piuvezam, M.R. Gamma-terpinene modulates acute inflammatory response in mice. Planta Med. 2015, 81, 1248-1254.

115. Fitsiou, E.; Anestopoulos, I.; Chlichlia, K.; Galanis, A.; Kourkoutas, I.; Panayiotidis, M.I.; Pappa, A. Antioxidant and antiproliferative properties of the essential oils of Satureja thymbra and Satureja parnassica and their major constituents. Anticancer Res. 2016, 36, 5757-5763. [CrossRef] [PubMed]

116. Kasuya, H.; Okada, N.; Kubohara, M.; Satou, T.; Masuo, Y.; Koike, K. Expression of BDNF and TH mRNA in the Brain Following Inhaled Administration of A-Pinene. Phytother. Res. 2015, 29, 43-47. [CrossRef] [PubMed]

117. Han, H.D.; Cho, Y.J.; Cho, S.K.; Byeon, Y.; Jeon, H.N.; Kim, H.S.; Kim, B.G.; Bae, D.S.; Lopez-Berestein, G.; Sood, A.K.; et al. Linalool-incorporated nanoparticles as a novel anticancer agent for epithelial ovarian carcinoma. Mol. Cancer Ther. 2016, 15, 618-627. [CrossRef]

118. Russo, E.B. Cannabis and epilepsy: An ancient treatment returns to the fore. Epilepsy Behav. 2017, 70, 292-297. [CrossRef] [PubMed]

119. Sulak, D.; Saneto, R.; Goldstein, B. The current status of artisanal Cannabis for the treatment of epilepsy in the United States. Epilepsy Behav. 2017, 70, 328-333. [CrossRef]

120. Piccinelli, A.C.; Santos, J.A.; Konkiewitz, E.C.; Oesterreich, S.A.; Formagio, A.S.; Croda, J.; Ziff, E.B.; Kassuya, C. Antihyperalgesic and antidepressive actions of (R)-(+)-Limonene, A-phellandrene, and essential oil from schinus terebinthifolius fruits in a neuropathic pain model. Nutr. Neurosci. 2015, 18, 24. [CrossRef]

121. Siqueira, H.D.; Neto, B.S.; Sousa, D.P.; Gomes, B.S.; da Silva, F.V.; Cunha, F.V.; Wanderley, C.W.; Pinheiro, G.; Cândido, A.G.; Wong, D.V.; et al. A-Phellandrene, a cyclic monoterpene, attenuates inflammatory response through neutrophil migration inhibition and mast cell degranulation. Life Sci. 2016, 160, 27-33. [CrossRef]

122. Lima, D.F.; Brandão, M.S.; Moura, J.B.; Leitão, J.M.; Carvalho, F.A.; Miúra, L.M.; Leite, J.R.; Sousa, D.P.; Almeida, F.R. Antinociceptive activity of the monoterpene $\alpha$-phellandrene in rodents: Possible mechanisms of action. J. Pharm. Pharmacol. 2012, 64, 283-292. [CrossRef] [PubMed]

123. Aydin, E.; Türkez, H.; Taşdemir, Ş. Anticancer and antioxidant properties of terpinolene in rat brain cells. Arch. Ind. Hyg. Toxicol. 2013, 64, 415-424. [CrossRef] [PubMed]

124. Turkez, H.; Aydın, E.; Geyikoglu, F.; Cetin, D. Genotoxic and oxidative damage potentials in human lymphocytes after exposure to terpinolene in vitro. Cytotechnology 2015, 67, 409-418. [CrossRef] [PubMed]

125. Paula-Freire, L.I.; Andersen, M.; Gama, V.; Molska, G.; Carlini, E.L. The oral administration of trans-caryophyllene attenuates acute and chronic pain in mice. Phytomedicine 2014, 21, 356-362. [CrossRef] [PubMed]

126. Varga, Z.V.; Matyas, C.; Erdelyi, K.; Cinar, R.; Nieri, D.; Chicca, A.; Nemeth, B.T.; Paloczi, J.; Lajtos, T.; Corey, L.; et al. Research Paper Themed Issue B-Caryophyllene protects against alcoholic steatohepatitis by attenuating inflammation and metabolic dysregulation in mice. Br. J. Pharmacol. 2017, 175, 320-334. [CrossRef] [PubMed]

127. Katsuyama, S.; Mizoguchi, H.; Kuwahata, H.; Komatsu, T.; Nagaoka, K.; Nakamura, H.; Bagetta, G.; Sakurada, T.; Sakurada, S. Involvement of peripheral cannabinoid and opioid receptors in $\beta$-caryophyllene-induced antinociception. Eur. J. Pain 2013, 17, 664-675. [CrossRef] [PubMed]

128. Xu, H.B.; Zheng, L.P.; Li, L.; Xu, L.Z.; Fu, J. Elemene, one ingredient of a chinese herb, against malignant tumors: A literature-based meta-analysis. Cancer Invest. 2013, 31, 156-166. [CrossRef] [PubMed] 
129. Yang, Q.; Wu, J.; Luo, Y.; Huang, N.; Zhen, N.; Zhou, Y.; Sun, F.; Li, Z.; Pan, Q.; Li, Y. (-)-Guaiol Regulates RAD51 stability via autophagy to induce cell apoptosis in non-small cell lung cancer. Oncotarget 2016, 7, 62585. [CrossRef] [PubMed]

130. Chinsembu, K.C. Tuberculosis and nature's pharmacy of putative anti-tuberculosis agents. Acta Tropica 2016, 153, 46-56. [CrossRef] [PubMed]

131. Semenya, S.; Potgieter, M.; Tshisikhawe, M.; Shava, S.; Maroyi, A. Medicinal utilization of exotic plants by bapedi traditional healers to treat human ailments in Limpopo province, South Africa. J. Ethnopharmacol. 2012, 144, 646-655. [CrossRef] [PubMed]

132. Yang, H.H.; Son, J.K.; Jung, B.; Zheng, M.; Kim, J.R. Epifriedelanol from the root bark of Ulmus davidiana inhibits cellular senescence in human primary cells. Planta Medica 2011, 77, 441-449. [CrossRef]

133. Zhou, X.; Wang, F.; Zhou, R.; Song, X.; Xie, M. Apigenin: A current review on its beneficial biological activities. J. Food Biochem. 2017, 41, e12376. [CrossRef]

134. He, M.; Min, J.W.; Kong, W.L.; He, X.H.; Li, J.X.; Peng, B.W. A review on the pharmacological effects of vitexin and isovitexin. Fitoterapia 2016, 115, 74-85. [CrossRef] [PubMed]

135. Sharma, A.; Kashyap, D.; Sak, K.; Tuli, H.S.; Sharma, A.K. Therapeutic charm of quercetin and its derivatives: A review of research and patents. Pharm. Pat. Anal. 2018, 7, 15-32. [CrossRef] [PubMed]

136. Ashaari, Z.; Hassanzadeh, G.; Alizamir, T.; Yousefi, B.; Keshavarzi, Z.; Mokhtari, T. The flavone luteolin improves central nervous system disorders by different mechanisms: A review. J. Mol. Neurosci. 2018, 65, 491-506. [CrossRef]

137. Rudroff, T.; Sosnoff, J.J. Cannabidiol to improve mobility in people with multiple sclerosis. Front. Neurol. 2018, 9, 183. [CrossRef] [PubMed]

138. Wong, K.U.; Baum, C.R. Acute Cannabis Toxicity. Pediatr. Emerg. Care 2019, 35, 799-806. [CrossRef] [PubMed]

139. International Conference on Harmonisation (ICH). All Guidelines. Available online: https://www.ich.org/page/ich-guidelines (accessed on 19 April 2020).

140. Schlag, A.K.; O'sullivan, S.E.; Zafar, R.R.; Nutt, D.J. Current controversies in medical cannabis: Recent developments in human clinical applications and potential therapeutics. Neuropharmacology 2021, 191, 108586. [CrossRef]

141. Hill, K.P. Cannabinoids and the coronavirus. Cannabis Cannabinoid Res. 2020, 5, 118-120. [CrossRef]

142. Costiniuk, C.T.; Jenabian, M.A. Acute inflammation and pathogenesis of SARS-CoV-2 infection: Cannabidiol as a potential anti-inflammatory treatment? Cytokine Growth Factor Rev. 2020, 53, 63. [CrossRef] [PubMed]

143. Byrareddy, S.N.; Mohan, M. SARS-CoV2 induced respiratory distress: Can cannabinoids be added to anti-viral therapies to reduce lung inflammation? Brain Behav. Immun. 2020, 87, 120. [CrossRef] [PubMed]

144. Esposito, G.; Pesce, M.; Seguella, L.; Sanseverino, W.; Lu, J.; Corpetti, C.; Sarnelli, G. The potential of cannabidiol in the COVID-19 pandemic. Br. J. Pharmacol. 2020, 177, 4967-4970. [CrossRef] [PubMed]

145. O'sullivan, S.E.; Stevenson, C.W.; Laviolette, S.R. Could cannabidiol be a treatment for coronavirus disease-19-related anxiety disorders? Cannabis Cannabinoid Res. 2021, 6, 7-18. [CrossRef] [PubMed] 